\title{
A lepto-hadronic model for high-energy emission from FR I radiogalaxies
}

\author{
M. M. Reynoso ${ }^{1}$, M. C. Medina $^{2}$, and G. E. Romero ${ }^{3}$ \\ ${ }^{1}$ Instituto de Investigaciones Físicas de Mar del Plata (CONICET - UNMdP), Facultad de Ciencias Exactas y Naturales, \\ Universidad Nacional de Mar del Plata, Dean Funes 3350, (7600) Mar del Plata, Argentina \\ 2 Irfu, Service de Physique des Particules, CEA Saclay, 91191 Gif-sur-Yvette Cedex, France \\ e-mail: clementina.medina@cea.fr \\ 3 Instituto Argentino de Radioastronomía, CCT La Plata-CONICET, 1894, Villa Elisa C.C. No. 5, Argentina \\ Received 17 May 2010 / Accepted 19 April 2011
}

\section{ABSTRACT}

\begin{abstract}
Context. The well known radiogalaxy Cen A has been recently detected as a source of very high energy (VHE) $\gamma$-rays by the HESS experiment just before Fermi/LAT detected it at high energies (HE). The detection, together with that of M 87, established radiogalaxies as VHE $\gamma$-ray emitters.

Aims. The aim of this work is to present a lepto-hadronic model for the VHE emission from the relativistic jets in FR I radiogalaxies. Methods. We consider that protons and electrons are accelerated in a compact region near the base of the jet, and they cool emitting multi wavelength radiation as propagating along the jet. The proton and electron distributions are obtained through steady-state transport equation taking into account acceleration, radiative and non-radiative cooling processes, as well as particle transport by convection.

Results. Considering the effects of photon absorption at different wavelengths, we calculate the radiation emitted by the primary protons and electrons, as well as the contribution of secondaries particles $\left(e^{ \pm}, \pi \mathrm{s}\right.$ and $\left.\mu \mathrm{s}\right)$. The expected high-energy neutrino signal is also obtained and the possibility of detections with KM3NeT and IceCube is discussed.

Conclusions. The spectral energy distribution obtained in our model with an appropriate set of parameters for an extended emission zone can account for much of the observed spectra for both AGNs.
\end{abstract}

Key words. astroparticle physics - radiation mechanisms: non-thermal - galaxies: individual: M 87 - galaxies: individual: Cen A

\section{Introduction}

The non-thermal high-energy emission from active galactic nuclei (AGNs) has been widely studied in recent years at different wavelength ranges through both satellite-borne and groundbased detectors (e.g. Urry \& Padovani 1995). Several theoretical models have been proposed to explain the electromagnetic emission of these objects. It is commonly accepted that the highenergy radiation is emitted by particles accelerated in the relativistic jets launched from the inner parts of an accretion disk that surrounds the central black hole. In general, the high-energy spectral energy distribution (SED) of AGNs presents two characteristic bumps. The lower energy bump, located at optical to X-ray energies, is usually explained as synchrotron emission of electrons while the origin of the high-energy peak in the SED is still under debate (see e.g. Böttcher 2007, for a review). Leptonic models attribute this component to inverseCompton up-scattering off synchrotron or external photons from the disk and/or radiation reprocessed in nearby clouds (see e.g. Katarzyński et al. 2001; Lenain et al. 2008; Ghisellini et al. 2005). In hadronic models, interactions of highly relativistic protons in the jet with ambient matter and photon fields, protoninduced cascades, or synchrotron radiation of protons, are responsible for the high-energy photons (see e.g. Mücke et al. 2000; Mücke \& Protheroe 2001; Reimer et al. 2004; Costamante et al. 2008; Hardcastle et al. 2009; Romero et al. 1996; Orellana $\&$ Romero 2009). There also exist models which are not based on the emission of accelerated particles in the relativistic jet and assume the production of $\mathrm{TeV} \gamma$-rays in a pulsar-like cascade mechanism in the magnetosphere of the black hole (e.g. Neronov \& Aharonian 2007; Rieger \& Aharonian 2008b).

The recently reported detection by HESS of the nearby radiogalaxy Cen A (Aharonian et al. 2009) is of great relevance since it establishes radiogalaxies as VHE $\gamma$-ray emitters. Cen $\mathrm{A}$ is the second non-blazar AGN discovered at VHE, after the HEGRA detection of $\gamma$-rays from M 87 (Aharonian et al. 2003) and the later confirmation by HESS (Aharonian et al. 2006). A great variety of leptonic and hadronic models has been already applied to this kind of sources and a full review is beyond the scope of this work. During the first year of operation, Fermi/LAT has detected HE emission from Centaurus A (Abdo et al. 2009a) and M 87 (Abdo et al. 2009c), providing new constraints to the models.

In this work we present a lepto-hadronic model for the emission from FR I radiogalaxies. Section 2 contains a brief description of observational facts on this type of sources. In Sect. 3 we present the outline of our scenario, describing its most relevant characteristics. Section 4 is devoted to the description of the model. In Sect. 5 we present the application to Centaurus A, whereas in Sect. 6 the results for M 87 are given. Finally, in Sect. 7 we discuss the model implications and perspectives.

\section{FR I radiogalaxies}

According to the unification model of AGNs (e.g. Urry \& Padovani 1995) FR I radiogalaxies, with their jet axis at a 
large angle with the line-of-sight, are the parent population of BL Lac objects whose jets are closely aligned to the line of sight. We concentrate here on the only two of them observed until now in the VHE range.

\subsection{Cen $A$}

Cen A is the closest FR I radiogalaxy ( $<4 \mathrm{Mpc}$, Harris et al. 1984; Hui et al. 1993) and its proximity makes it uniquely observable among such objects, eventhough its bolometric luminosity is not high as compared to other AGNs. It is very active at radio wavelengths presenting a rich jet structure. We can distinguish in its structure two components: inner jets at a kpc scale and giants lobes covering $10^{\circ}$ in the sky. A detailed description of the radio morphology can be found in Meier et al. (1989). The inner kpc jet has also been detected in X-rays (Kraft et al. 2002) with an structure of knots and diffuse emission. Recently Croston et al. (2009) reported the detection of non-thermal X-ray emission from the shock of the southwest inner radio lobe from deep Chandra observations.

The supermassive black hole at the center of the active galaxy has an estimated mass of about $10^{7} M_{\odot}$ (Neumayer et al. 2007; Cappellari et al. 2009; Israel 1998) to $10^{8} M_{\odot}$ (Silge et al. 2005; Marconi et al. 2001). The black hole host galaxy is an elliptical one (NGC 5128) with a twisted disk which obscures the central engine at optical wavelengths.

Cen A was observed by the Compton Gamma Ray Observatory (CGRO) with all its instruments from $\mathrm{MeV}$ to GeV energies (Gehrels \& Cheung 1992; Kinzer et al. 1995; Paciesas et al. 1993; Steinle et al. 1998; Thompson et al. 1995). In this period, this source exhibited X-ray variability (Bond et al. 1996) and also some soft $\gamma$-ray variability (Bond et al. 1996; Kinzer et al. 1995; Steinle et al. 1998). However, Sreekumar et al. (1999) found that the EGRET flux was stable during the whole period of CGRO observations.

In 1999 the new Chandra X-ray Observatory took images of Cen A with an unprecedented resolution. More than 200 X-ray point sources were identified in those images (Kraft et al. 2001).

Cen A as a possible source of UHE cosmic rays was early proposed by (Romero et al. 1996). Recently, the Pierre Auger Collaboration reported the existence of anisotropy on the arrival directions of UHE cosmic rays (Abraham et al. 2007), remarking that at least 2 of this events can be correlated with the Cen A position $\left(3^{\circ}\right.$ circle). Further works have claimed that there are several events that can be associated with Cen $\mathrm{A}$ and its big radio lobes (Gorbunov et al. 2008; Fargion 2008; Wibig \& Wolfendale 2007) but this correlation is still statistically weak.

Finally, Fermi/LAT has detected Cen A in the first three months of survey with a significance above $10 \sigma$ (Abdo et al. 2009b).

\section{2. $M 87$}

The giant radiogalaxy M 87 is located at $16.7 \mathrm{Mpc}$ within the Virgo cluster (Macri et al. 1999). It presents a one-sided jet which is inclined with respect to the line of sight an angle between $20^{\circ}-40^{\circ}$ (Biretta et al. 1995, 1999). In addition to its bright and well resolved jet, $\mathrm{M} 87$ harbors a very massive black hole $(6.0 \pm 0.5) \times 10^{9} M_{\odot}($ Gebhardt \& Thomas 2009) which is thought to power the relativistic outflow. Given its proximity, the substructures inside the jet could be resolved in the X-ray, optical, and radio wavebands (Wilson \& Yang 2002). High frequency VLBI observations have resolved the inner jet up to about 70 Schwarzschild radii (Junor et al. 1999). Along the jet, nearly stationary components (Marscher et al. 2008) and features moving at superluminal speeds (Ly et al. 2007; Kovalev et al. 2007) were observed (100 pc-scale).

M 87 is also a well-known VHE $\gamma$-rays emitter (Aharonian et al. 2003, 2006; Acciari et al. 2008; Albert et al. 2008) showing a $\gamma$-ray flux variability on short time scales with flaring phenomena in VHE, radio, and X-ray wavebands simultaneously (Acciari et al. 2009). Recently, it was detected by Fermi/LAT with a significance greater than $10 \sigma$ in 10 months of observations (Abdo et al. 2009c).

Rapid variability constrains the emission region extent to less than $\approx 5 \delta R_{\mathrm{S}}$, where $\delta$ is the relativistic Doppler factor. Some suggested explanations for the VHE $\gamma$-ray emission were ruled out (e.g. dark matter annihilation, Baltz et al. 2000). At the same time, various VHE $\gamma$-ray jet emission models were proposed: leptonic (Georganopoulos et al. 2005; Lenain et al. 2008) and hadronic (Reimer et al. 2004) ones. However, the location of the emission region is still unknown. The nucleus (Neronov \& Aharonian 2007; Rieger \& Aharonian 2008a), the inner jet (Tavecchio \& Ghisellini 2008), or larger structures in the jet such as the knot HST-1, have been discussed as possible sites of particle acceleration (Cheung et al. 2007).

\section{Basic scenario}

We assume that a population of relativistic particles can be accelerated to very high energies close to the base of the AGN jet. These primary electrons and protons carry a fraction of the total kinetic power of the jet $L_{\mathrm{j}}^{(\mathrm{kin})}$, and as they are dragged along with the jet, they cool giving rise to electromagnetic emission and neutrinos.

Assuming that a fraction $q_{\mathrm{j}}$ of the Eddington luminosity is carried by the jet and a counter jet, the jet kinetic power is

$L_{\mathrm{j}}^{(\mathrm{kin})}=\frac{q_{\mathrm{j}}}{2} \frac{4 \pi G M_{\mathrm{bh}} m_{\mathrm{p}} c}{\sigma_{\mathrm{T}}}$.

This power can be very high if the jet is launched by a dissipationless accretion disk (Bogovalov \& Kelner 2010).

Most of the jet content is in the form of a thermal plasma with a constant bulk Lorentz factor $\Gamma_{\mathrm{b}}$. This plasma is initially in equipartition with a tangled magnetic field at the Alfvén surface $\left(z_{0}=50 R_{\mathrm{g}}\right.$ from the central black hole) (e.g. McKinney 2006). The highly disorganized magnetic field has a root mean square value $B(z)$ at a distance $z$ from the black hole in the observer frame, such that $B^{2}(z):=\left\langle B_{x}^{2}\right\rangle+\left\langle B_{y}^{2}\right\rangle+\left\langle B_{z}^{2}\right\rangle$. The magnetic energy density for $z=z_{0}$ is then $\rho_{\mathrm{m}}\left(z_{0}\right)=B_{0}^{2} /(8 \pi)$ with $B_{0}=B\left(z_{0}\right)$. Equating the magnetic to the kinetic energy density, yields:

$B_{0}=\sqrt{\frac{8 L_{\mathrm{j}}^{(\mathrm{kin})}}{\left[r_{\mathrm{j}}\left(z_{0}\right)\right]^{2} v_{\mathrm{b}}}}$,

where $z_{0}$ is the distance to the black hole, $v_{\mathrm{b}}$ is the jet velocity, and $r_{\mathrm{j}}\left(z_{0}\right)=z_{0} \tan \xi_{\mathrm{j}}$ is the radius of the jet assuming that it has a conical shape with half-opening angle $\xi_{j}$. A widely accepted view is that jets are accelerated through the conversion of magnetic energy into kinetic energy (e.g. Komissarov et al. 2007). We adopt a phenomenological dependence on the distance to the black hole for the magnetic field (e.g. Krolik 1999),

$B(z)=B_{0}\left(\frac{z_{0}}{z}\right)^{m}$. 
Since the density of cold material within the jet decays as $z^{-2}$, using an exponent $m \in(1,2)$ in the above expression implies that, as $z$ increases, the magnetic energy decreases more rapidly than the kinetic one. The corresponding increase in the bulk Lorentz factor is taken into account as described in Appendix A. In the following, we will write simply $\Gamma_{b}$, but it actually depends on $z$.

The particle acceleration takes place in a compact but inhomogeneous region of size $\Delta z<z_{\text {acc }}$ near the base of the jet, at a distance $z_{\text {acc }}$ away from the black hole. The value of $z_{\text {acc }}$ is fixed by requiring the magnetic energy density to be in subpartition with the jet kinetic energy density. This condition enables strong shocks to develop (Gaisser 1990). Assuming that at $z_{\text {acc }}$ the magnetic energy is a fraction $q_{\mathrm{m}}$ of its value at $z_{0}$, it follows that

$z_{\mathrm{acc}}=z_{0} q_{\mathrm{m}}^{\frac{1}{2-2 m}}$

For example, if $M_{\mathrm{bh}}=10^{8} M_{\odot}, m=1.5$, and $z_{0}=50 R_{\mathrm{g}}$, using $q_{\mathrm{m}}=0.38$ yields a distance $z_{\mathrm{acc}}=132 R_{\mathrm{g}}$.

The power injected in the form of relativistic particles $\left(L_{\mathrm{rel}}\right)$ is considered to be a small fraction $q_{\text {rel }}$ of the total jet kinetic power and the relation between proton and electron powers is given by the parameter $a$ such that $L_{\mathrm{p}}=a L_{\mathrm{e}}$ (see e.g. Romero \& Vila 2008; Vila \& Romero 2010).

These parameters are constrained by the available observational data on each source and reasonable theoretical considerations. In the following section, we describe the procedure used to obtain the particle distributions along the jet and the radiative output that then arises.

The existence of heavy jets magnetically driven in AGN and microquasars has been supported by several scientific works in the last few years. For example, Sa̧dowski \& Sikora (2010) conclude that mildly relativistic proton-electron jets might be formed by magnetocentrifugal launching by inner portion of magnetized disks around rotating black holes. This leads to a triple-component jet structure: proton-electron component sourrounded by the relativistic pair-dominated sheat. The final speed of this centrifugal outflow depends strongly on the disk vertical structure (see e.g. Wardle \& Koenigl 1993) which is certainly unknown. However, for barionic outflows to reach mildly relativistic speeds, some inital boost would be neccesary, which would be produced by heating or mechanically, by flaring activity and/or by radiation pressure. Previous work by Heinz (2008) has proved the existence of a relativistic and baryon-loaded jet in Cygnus X-1 and that the bulk of the kinetic energy is carried by cold protons, as in the case of SS 433. For further information about magnetically launched jets see (Spruit 2010), where the production, acceleration, collimation, and composition of jets are well explained.

\section{Description of the model}

The model developed for this work is based on the energy distribution of the different particle populations along the jet. These are obtained as solutions of a 1-dimensional steady-state transport equation that includes the relevant cooling terms and a convective one. The radiative output is obtained in the jet reference frame, where the particle distributions are isotropic, and the result is transformed back to the observer frame.

The procedure begins with the calculation of the distribution of primary electrons along the jet taking into account synchrotron and adiabatic cooling. After that, the synchrotron radiation emitted by the primary electrons can be calculated. To check that the electron distribution is consistent with the energy loss mechanisms operating, the synchrotron cooling rate must be much greater than the inverse Compton (IC) one due to electrons interacting with the synchrotron photons (SSC). If this is the case, it means that the main cooling is due to synchrotron radiation, and neglecting the IC energy loss is a valid approximation to obtain the electron distribution. If the SSC cooling can not be neglected, then the transport equation becomes more complicated and a different approach is needed (e.g. Schlickeiser 2009).

Having obtained the electron distribution, the next step is to calculate the distribution of primary protons taking into account the cooling due to synchrotron emission, adiabatic expansion, $p p$ and $p \gamma$ interactions. The latter two types of interactions yield the production of secondary pions, muons, and electronpositron pairs. These three populations of particles are also described with the transport equation, and the radiative output that they produce is also considered. According to, e.g. Khangulyan et al. (2008) and Pellizza et al. (2010), it can be seen that in the present scenario, IC cascades are suppressed by the synchrotron cooling of secondary $e^{ \pm}$, since the magnetic field is greater than $10 \mathrm{G}$ in the regions of the jet where emission takes place. Therefore, we neglect the effect of IC cascading and calculate the synchrotron emission of the secondary electrons and positrons.

In this section we present all the relevant expressions used in this model. We discuss on the injection of primary particles, the relevant cooling rates, the transport equation used, the injection of secondary particles, and the emission of photons and neutrinos.

\subsection{Injection of primary particles}

At a distance $z$ to the black hole taken in the reference frame of the observer, we adopt an injection function in terms of the particle energy $E^{\prime}$ in the co-moving reference frame of the jet:

$$
\begin{aligned}
Q_{i}^{\prime}\left(E^{\prime}, z\right) & :=\frac{\mathrm{d} \mathcal{N}_{i}}{\mathrm{~d} E^{\prime} \mathrm{d} V^{\prime} \mathrm{d} \Omega^{\prime} \mathrm{d} t^{\prime}} \quad\left(\mathrm{GeV}^{-1} \mathrm{~s}^{-1} \mathrm{~cm}^{-3} \mathrm{sr}^{-1}\right) \\
& =K_{i}\left(\frac{z_{\mathrm{acc}}}{z}\right)^{2} E^{\prime-s} \exp \left[-\left(\frac{E^{\prime}}{{E^{\prime}}_{i}^{(\mathrm{max})}}\right)\right] .
\end{aligned}
$$

Here, $\mathcal{N}_{i}$ represents the total number of relativistic electrons $(i=e)$ or protons $(i=p)$, and the cut-off energy is obtained from the balance of particle gains and losses. These processes are described below.

The injection function can be transformed to the observer frame by taking into account that

$$
\frac{E^{\prime}}{p^{\prime 2}} \frac{\mathrm{d} \mathcal{N}_{i}}{\mathrm{~d} V^{\prime} \mathrm{d} p^{\prime} \mathrm{d} \Omega^{\prime} \mathrm{d} t^{\prime}}
$$

is a Lorentz invariant (e.g. Dermer \& Schlickeiser 2002; Torres \& Reimer 2011). From this, it follows that the particle injection in the observer frame is given by

$Q_{i}(E, z)=\left[\frac{E^{2}-m_{i}^{2} c^{4}}{E^{\prime 2}-m_{i}^{2} c^{4}}\right]^{1 / 2} Q_{i}^{\prime}\left(E^{\prime}, z^{\prime}\right)$,

where the energy in the jet frame is given in terms of the energy in the observer frame $E$, and the angle $\theta$ between the particle momentum $\boldsymbol{p}$ and the bulk velocity of the jet $\boldsymbol{v}_{\mathrm{b}}=c \beta_{\mathrm{b}} \hat{z}$ :

$E^{\prime}=\Gamma_{\mathrm{b}}\left(E-\beta_{\mathrm{b}} \cos \theta \sqrt{E^{2}-m_{i}^{2} c^{4}}\right)$. 
The normalization constant $K_{i}$ is found in each case using the power in relativistic species $L_{\{e, p\}}$, integrating in the energy, solid angle, and volume of the acceleration zone $\left(z \in\left[z_{\text {acc }}, z_{\mathrm{acc}}+\Delta z\right]\right)$ :

$L_{i}=\int_{\Delta E} \mathrm{~d} E \int_{4 \pi} \mathrm{d} \Omega \int_{\Delta V} \mathrm{~d} V E Q_{i}(E, z)$.

\subsection{Accelerating and cooling processes}

To determine the maximum energies, it is necessary to account for the particles acceleration and cooling rates $t^{\prime-1}=$ $E^{\prime-1}\left|\mathrm{~d} E^{\prime} / \mathrm{d} t^{\prime}\right|$.

We assume that in the acceleration zone, particles are accelerated by a diffusive shock-modulated mechanism with a rate (e.g. Begelman et al. 1990):

$t_{\text {acc }}^{\prime-1}\left(E^{\prime}, z\right)=\eta \frac{c e B^{\prime}(z)}{E^{\prime}}$

where $0<\eta \ll 1$ is the efficiency of the mechanism. The magnetic field in the jet frame is assumed to be random and with no preferred direction. $B^{\prime}$ represents the root mean square field in the jet frame: $B^{\prime 2}=\left\langle B_{x^{\prime}}^{\prime 2}\right\rangle+\left\langle B_{y^{\prime}}^{\prime}\right\rangle+\left\langle B_{z^{\prime}}^{\prime}{ }^{2}\right\rangle$. As considered also in Heinz \& Begelman (2000), the average of each component of the magnetic field is supposed to vanish in the jet frame: $\left\langle B_{i}^{\prime}\right\rangle=0$. Assuming that there is no electric field in the jet frame, the components of the magnetic field in the observer frame are related to the ones in the jet frame as $B_{x}=\Gamma_{\mathrm{b}} B_{x^{\prime}}^{\prime}$, $B_{y}=\Gamma_{\mathrm{b}} B_{y^{\prime}}^{\prime}$, and $B_{z}=B_{z^{\prime}}^{\prime}$, which yields ${ }^{1}$

$B^{\prime}(z)=\sqrt{\frac{3}{2 \Gamma_{\mathrm{b}}^{2}+1}} B(z)$

where $B(z)$ is given by Eq. (3).

As for the energy loss processes, particles emit synchrotron radiation at a rate

$t_{\text {sync }}^{\prime-1}\left(E^{\prime}, z\right)=\frac{4}{3}\left(\frac{m_{\mathrm{e}}}{m}\right)^{3} \frac{\sigma_{\mathrm{T}} B^{\prime 2}}{m_{\mathrm{e}} c 8 \pi} \frac{E^{\prime}}{m c^{2}}$.

The lateral expansion of the jet implies an adiabatic cooling rate (Bosch-Ramon et al. 2006) in the observer frame given by

$t_{\mathrm{ad}}^{-1}(E, z)=\frac{2}{3} \frac{v_{\mathrm{b}}}{z}$

The density of cold matter at a distance $z$ from the black hole is, in observer frame,

$n_{\mathrm{c}}(z)=\frac{\left(1-q_{\mathrm{rel}}\right) \dot{m}_{\mathrm{j}}}{m_{\mathrm{p}} \pi z^{2} \tan ^{2} \xi_{\mathrm{j}} v_{\mathrm{b}}}$,

where the mass loss rate in the jet is

$\dot{m}_{\mathrm{j}}=\left.\frac{L_{\mathrm{j}}^{(\mathrm{kin})}\left(\Gamma_{\mathrm{b}}-1\right)}{c^{2}}\right|_{z_{0}}$

In the jet frame, the cold matter density is

$n_{\mathrm{c}}^{\prime}(z)=\frac{n_{\mathrm{c}}(z)}{\Gamma_{\mathrm{b}}}$

\footnotetext{
${ }^{1}$ Note that Eq. (12) is consistent with Eq. (3) of (Heinz \& Begelman 2000) if the radial component of their flow velocity can be neglected.
}

Relativistic protons in the jet undergo $p p$ collisions with these cold protons at a rate

$t_{p p}^{\prime-1}\left(E^{\prime}, z\right)=n_{\mathrm{c}}^{\prime}(z) c \sigma_{p p}^{(\mathrm{inel})}\left(E^{\prime}\right) K_{p p}$.

Here the inelasticity coefficient is $K_{p p} \approx 1 / 2$, the corresponding cross section for inelastic $p p$ interactions can be approximated by (Kelner et al. 2006, 2009)

$$
\begin{aligned}
\sigma_{p p}^{(\text {inel })}\left(E_{\mathrm{p}}\right)= & \left(34.3+1.88 L+0.25 L^{2}\right) \\
& \times\left[1-\left(\frac{E_{\mathrm{th}}}{E_{\mathrm{p}}}\right)^{4}\right]^{2} \times 10^{-27} \mathrm{~cm}^{2},
\end{aligned}
$$

where $L=\ln \left(E_{\mathrm{p}} / 1000 \mathrm{GeV}\right)$ and $E_{\mathrm{th}}=1.2 \mathrm{GeV}$.

\subsubsection{Synchrotron radiation and Inverse Compton interactions}

To consider IC interactions of primary electrons with synchrotron photons in the jet (SSC), it is necessary to know the particle distribution of the synchrotron emitting particles, $N_{\mathrm{e}, \mathrm{p}}^{\prime}\left(E^{\prime}, z\right)$, which is obtained by solving the transport equation described below. Actually, the synchrotron emission of electrons dominates the low energy photon background which is a good target for IC and $p \gamma$ interactions. Since in the cases considered here, IC cooling is much less efficient than synchrotron cooling, the electron distribution can be obtained to a good approximation without considering IC cooling. In the jet frame, the background radiation density, in units $\left[\mathrm{GeV}^{-1} \mathrm{~cm}^{-3}\right]$, has been approximated locally as

$n_{\mathrm{ph}}^{\prime}\left(E_{\mathrm{ph}}^{\prime}, z\right) \approx \frac{\varepsilon_{\mathrm{syn}}^{\prime}\left(E_{\mathrm{ph}}^{\prime}, z\right)}{E_{\mathrm{ph}}^{\prime}} \frac{r_{\mathrm{j}}(z)}{c}$,

where $\varepsilon_{\text {syn }}^{\prime}$ is the power per unit energy per unit volume of the synchrotron photons,

$\varepsilon_{\mathrm{syn}}^{\prime}\left(E_{\mathrm{ph}}^{\prime}, z\right)=\left(\frac{1-e^{-\tau_{\mathrm{SSA}}\left(E_{\mathrm{ph}}^{\prime}, z\right)}}{\tau_{\mathrm{SSA}}\left(E_{\mathrm{ph}}^{\prime}, z\right)}\right) \int_{m_{\mathrm{e}} c^{2}}^{\infty} \mathrm{d} E^{\prime} 4 \pi P_{\mathrm{syn}} N_{\mathrm{e}}^{\prime}\left(E^{\prime}, z\right)$.

Here, the synchrotron power per unit energy emitted by the electrons is given by (Blumenthal \& Gould 1970):

$P_{\text {syn }}\left(E_{\mathrm{ph}}^{\prime}, E^{\prime}, z\right)=\frac{\sqrt{2} e^{3} B^{\prime}(z)}{m_{\mathrm{e}} c^{2} h} \frac{E_{\mathrm{ph}}^{\prime}}{E_{\mathrm{cr}}} \int_{E_{\mathrm{ph}}^{\prime} / E_{\mathrm{cr}}}^{\infty} \mathrm{d} \zeta K_{5 / 3}(\zeta)$,

where $K_{5 / 3}(\zeta)$ is the modified Bessel function of order $5 / 3$ and

$E_{\mathrm{cr}}=\frac{\sqrt{6} h e B^{\prime}(z)}{4 \pi m_{\mathrm{e}} c}\left(\frac{E^{\prime}}{m_{\mathrm{e}} c^{2}}\right)^{2}$.

The factor in parenthesis in Eq. (19) accounts for the effect of synchrotron self-absorption (SSA) within the jet, with an optical depth

$\tau_{\mathrm{SSA}}\left(E_{\mathrm{ph}}^{\prime}, z\right)=\int_{0}^{\frac{r_{\mathrm{j}}(z)}{\sin \theta^{\prime}}} \mathrm{d} l^{\prime} \alpha_{\mathrm{SSA}}\left(E_{\mathrm{ph}}^{\prime}, z^{\prime}\left(l^{\prime}\right)\right)$,

where $\theta^{\prime}$ is such that $\tan \theta^{\prime}=\Gamma_{\mathrm{b}} \tan \theta$, and the SSA coefficient is given by (Rybicki \& Lightman 1979):

$$
\begin{aligned}
\alpha_{\mathrm{SSA}}= & -\frac{h^{3} c^{2}}{8 \pi E_{\mathrm{ph}}^{\prime 2}} \int_{m_{\mathrm{e}} c^{2}}^{\infty} \mathrm{d} E^{\prime} E^{\prime 2} P_{\mathrm{syn}}\left(E_{\mathrm{ph}}^{\prime}, E^{\prime}\right) \\
& \times \frac{\partial}{\partial E^{\prime}}\left[\frac{N_{\mathrm{e}}^{\prime}\left(E^{\prime}, z^{\prime}\left(l^{\prime}\right)\right)}{E^{\prime 2}}\right] .
\end{aligned}
$$


After all these considerations, the cooling rate due to IC scattering for electrons of energy $E^{\prime}$ in the jet frame can be obtained by integrating in the target photon energy $E_{\mathrm{ph}}^{\prime}$ and in energy of the scattered photon $E_{\gamma}^{\prime}$ (Blumenthal \& Gould 1970):

$$
\begin{aligned}
t_{\mathrm{IC}}^{\prime-1}\left(E^{\prime}, z\right)= & \frac{3 m_{\mathrm{e}}^{2} c^{4} \sigma_{\mathrm{T}}}{4 E^{\prime 3}} \int_{E_{\mathrm{ph}}^{\prime(\min )}}^{E^{\prime}} \mathrm{d} E_{\mathrm{ph}}^{\prime} \frac{n_{\mathrm{ph}}^{\prime}\left(E_{\mathrm{ph}}^{\prime}, z\right)}{E_{\mathrm{ph}}^{\prime}} \\
& \times \int_{E_{\mathrm{ph}}^{\prime}}^{\frac{\Gamma_{\mathrm{e}}}{\Gamma_{\mathrm{e}}+1} E^{\prime}} \mathrm{d} E_{\gamma}^{\prime} F(q)\left[E_{\gamma}^{\prime}-E_{\mathrm{ph}}^{\prime}\right],
\end{aligned}
$$

where $E_{\mathrm{ph}}^{\prime(\mathrm{min})}$ is the lowest energy of the available background of synchrotron photons, and

$F(q)=2 q \ln q+(1+2 q)(1-q)+\frac{1}{2}(1-q) \frac{\left(q \Gamma_{\mathrm{e}}^{\prime}\right)^{2}}{1+\Gamma_{\mathrm{e}}^{\prime}}$,

with $\Gamma_{\mathrm{e}}^{\prime}=4 E_{\mathrm{ph}}^{\prime} E^{\prime} /\left(m_{\mathrm{e}}^{2} c^{4}\right)$ and

$q=\frac{E_{\gamma}^{\prime}}{\Gamma_{\mathrm{e}}^{\prime} E_{\mathrm{ph}}^{\prime}\left(1-E_{\gamma}^{\prime} / E_{\mathrm{ph}}^{\prime}\right)}$.

\subsubsection{Proton-photon interactions}

Proton interactions with the photon background can be an important cooling process, with a rate appoximated as

$$
\begin{aligned}
t_{\mathrm{p} \gamma}^{\prime-1}\left(E^{\prime}, z\right)= & \frac{c}{2 \gamma_{\mathrm{p}}^{2}} \int_{\frac{\epsilon_{\mathrm{th}}}{2 \gamma_{\mathrm{p}}}}^{\infty} \mathrm{d} E_{\mathrm{ph}}^{\prime} \frac{n_{\mathrm{ph}}^{\prime}\left(E_{\mathrm{ph}}^{\prime}, z\right)}{E_{\mathrm{ph}}^{\prime 2}} \\
& \times \int_{\epsilon_{\mathrm{th}}^{(\pi)}}^{2 E_{\mathrm{ph}}^{\prime} \gamma_{\mathrm{p}}} \mathrm{d} \epsilon_{\mathrm{r}} \sigma_{\mathrm{p} \gamma}^{(\pi)}\left(\epsilon_{\mathrm{r}}\right) K_{\mathrm{p} \gamma}^{(\pi)}\left(\epsilon_{\mathrm{r}}\right) \epsilon_{\mathrm{r}} .
\end{aligned}
$$

Here, $\epsilon_{\mathrm{th}}^{(\pi)}=150 \mathrm{MeV}$ and we use the expressions for the cross section $\sigma_{\mathrm{p} \gamma}^{(\pi)}$ and the inelasticity $K_{\mathrm{p} \gamma}^{(\pi)}$ given in Begelman et al. (1990).

\subsection{Particle distributions in the jet}

The energy distribution for each particle population is obtained as a solution to the following 1D stationary transport equation (e.g. Ginzburg \& Syrovatskii 1964; Khangulyan et al. 2008):

$v_{\mathrm{b}} \frac{\partial N^{\prime}\left(E^{\prime}, z\right)}{\partial z}+\frac{\partial\left[b\left(E^{\prime}, z\right) N^{\prime}\left(E^{\prime}, z\right)\right]}{\partial E^{\prime}}+\frac{N^{\prime}\left(E^{\prime}, z\right)}{T_{\mathrm{d}}\left(E^{\prime}\right)}=Q^{\prime}\left(E^{\prime}, z\right)$.

This equation includes the effects of convection with a speed $v_{\mathrm{b}} \sim c$, and particle cooling with an energy loss $b\left(E^{\prime}, z\right)=$ $\mathrm{d} E^{\prime} / \mathrm{d} t$. Particle decay with a timescale $T_{\mathrm{d}}\left(E^{\prime}\right)$ is also considered in the cases of secondary pions and muons. We note that the energy $E^{\prime}$ in the above equation corresponds to the co-moving frame, where the particle distributions are isotropic, while the spatial coordinate $z$ corresponds to the observer frame (e.g. Jokipii \& Parker 1970; Kirk et al. 1988). The source term is given by a function $Q^{\prime}\left(E^{\prime}, z\right)$, which in the case of primary particles is given by Eq. (6), while for secondaries it is obtained using the parent particle distribution $\left(N^{\prime}\left(E^{\prime}, z\right)\right)$ along with the secondary particle production rates (see Sect. 4.3.1).

We can solve the transport equation using the method of characteristics, i.e., writing

$$
\frac{\mathrm{d} z}{v_{\mathrm{b}}}=\frac{\mathrm{d} E^{\prime}}{b\left(E^{\prime}, z\right)}=\frac{\mathrm{d} N^{\prime}\left(E^{\prime}, z\right)}{Q^{\prime}\left(E^{\prime}, z\right)-\left[\frac{1}{T_{\mathrm{d}}\left(E^{\prime}\right)}+\frac{\partial b\left(E^{\prime}, z\right)}{\partial E}\right] N(E, z)},
$$

where the first two terms allow us to find a characteristic curve $z_{\mathrm{c}}\left(E_{\mathrm{c}}\right)$ for each pair $\left(E^{\prime}, z\right)$ of interest. Equating the second and third members it follows that

$$
\begin{aligned}
N^{\prime}\left(E^{\prime}, z\right)= & \int_{E}^{\infty} \mathrm{d} E_{\mathrm{c}} \frac{Q^{\prime}\left(E_{\mathrm{c}}, z_{\mathrm{c}}\right)}{\left|b\left(E_{\mathrm{c}}, z_{\mathrm{c}}\right)\right|} \\
& \times \exp \int_{E^{\prime}}^{E_{\mathrm{c}}} \mathrm{d} E^{\prime \prime}\left[\frac{T_{\mathrm{d}}\left(E^{\prime \prime}\right)\left|\frac{\mathrm{d} b}{\mathrm{~d} E}\left(E^{\prime \prime}, z^{\prime \prime}\right)\right|-1}{T_{\mathrm{d}}\left(E^{\prime \prime}\right)\left|b\left(E^{\prime \prime}, z^{\prime \prime}\right)\right|}\right] .
\end{aligned}
$$

\subsubsection{Pion and muon production}

As high energy protons interact with background matter and radiation, they produce pions.

The pion injection due to $p p$ interactions in the jet frame is calculated as

$$
\begin{aligned}
Q_{\pi, p p}^{\prime}\left(E_{\pi}^{\prime}, z\right)= & n_{\mathrm{c}}^{\prime}(z) c \int_{0}^{1} \frac{\mathrm{d} x}{x} N_{\mathrm{p}}^{\prime}\left(\frac{E_{\pi}^{\prime}}{x}, z\right) \\
& \times F_{\pi}\left(x, \frac{E_{\pi}^{\prime}}{x}\right) \sigma_{p p}^{\text {(inel) }}\left(\frac{E_{\pi}^{\prime}}{x}\right)
\end{aligned}
$$

where

$$
\begin{aligned}
F_{\pi}\left(x, \frac{E_{\pi}^{\prime}}{x}\right)= & 4 \alpha B_{\pi} x^{\alpha-1}\left(\frac{1-x^{\alpha}}{1+r^{\prime} x^{\alpha}\left(1-x^{\alpha}\right)}\right)^{4} \\
& \times\left(\frac{1}{1-x^{\alpha}}+\frac{r^{\prime}\left(1-2 x^{\alpha}\right)}{1+r^{\prime} x^{\alpha}\left(1-x^{\alpha}\right)}\right)\left(1-\frac{m_{\pi} c^{2}}{E_{\pi}^{\prime}}\right)^{1 / 2}
\end{aligned}
$$

is the distribution of pions produced per $p p$ collision, with $x=$ $E_{\pi}^{\prime} / E^{\prime}, B_{\pi}=a^{\prime}+0.25, a^{\prime}=3.67+0.83 L+0.075 L^{2}, r^{\prime}=2.6 / \sqrt{a^{\prime}}$, and $\alpha=0.98 / \sqrt{a^{\prime}}$ (see Kelner et al. 2006, 2009).

In the same way, the source function for charged pions produced by $p \gamma$ interactions is

$$
\begin{aligned}
Q_{\pi, p \gamma}^{\prime}\left(E_{\pi}^{\prime}, z\right)= & \int_{E_{\pi}^{\prime}} \mathrm{d} E^{\prime} N_{\mathrm{p}}^{\prime}\left(E^{\prime}, z\right) \omega_{\mathrm{p} \gamma}^{\prime(\pi)}\left(E^{\prime}, z\right) \\
& \times \mathcal{N}_{\pi}\left(E^{\prime}\right) \delta\left(E_{\pi}^{\prime}-0.2 E^{\prime}\right) \\
= & 5 N_{\mathrm{p}}^{\prime}\left(5 E_{\pi}^{\prime}, z\right) \omega_{\mathrm{p} \gamma}^{\prime(\pi)}\left(5 E_{\pi}^{\prime}, z\right) \mathcal{N}_{\pi}\left(5 E_{\pi}^{\prime}\right) .
\end{aligned}
$$

Here $\omega_{\mathrm{p} \gamma}^{\prime(\pi)}$ is the $p \gamma$ collision frequency defined as (Atoyan \& Dermer 2003):

$$
\begin{aligned}
\omega_{\mathrm{p} \gamma}^{\prime(\pi)}\left(E^{\prime}, z\right)= & \frac{c}{2 \gamma_{\mathrm{p}}^{2}} \int_{\frac{\epsilon_{\mathrm{th}}}{2 \gamma_{\mathrm{p}}}}^{\infty} \mathrm{d} E_{\mathrm{ph}}^{\prime} \frac{n_{\mathrm{ph}}^{\prime}\left(E_{\mathrm{ph}}^{\prime}, z\right)}{E_{\mathrm{ph}}^{\prime 2}} \\
& \times \int_{\epsilon_{\mathrm{th}}^{(\pi)}}^{2 \epsilon \gamma_{\mathrm{p}}} \mathrm{d} \epsilon^{\prime} \sigma_{\mathrm{p} \gamma}^{(\pi)}\left(\epsilon^{\prime}\right) \epsilon^{\prime},
\end{aligned}
$$

and the mean number of positive or negative pions is

$\mathcal{N}_{\pi} \approx \frac{p_{1}}{2}+2 p_{2}$.

This number depends on the probabilities of single pion and multi-pion production $p_{1}$ and $p_{2}=1-p_{2}$. Using the mean inelasticity function $\bar{K}_{\mathrm{p} \gamma}=t_{\mathrm{p} \gamma}^{-1} / \omega_{\mathrm{p} \gamma}^{(\pi)}$, the probability $p_{1}$ is

$p_{1}=\frac{K_{2}-\bar{K}_{\mathrm{p} \gamma}}{K_{2}-K_{1}}$

where $K_{1}=0.2$ and $K_{2}=0.6$.

The injection functions of charged pions given by Eqs. (28), (30) are used to work out the distribution $N_{\pi}^{\prime}\left(E^{\prime}, z\right)$ by solving the transport Eq. (25). 
For muon injection, we proceed as (Lipari et al. 2007) and we consider the production of left handed and right handed muons separately, which have different decay spectra:

$\frac{\mathrm{d} n_{\pi^{-} \rightarrow \mu_{L}^{-}}}{\mathrm{d} E_{\mu}}\left(E_{\mu} ; E_{\pi}\right)=\frac{r_{\pi}(1-x)}{E_{\pi} x\left(1-r_{\pi}\right)^{2}} \Theta\left(x-r_{\pi}\right)$
$\frac{\mathrm{d} n_{\pi^{-} \rightarrow \mu_{R}^{-}}}{\mathrm{d} E_{\mu}}\left(E_{\mu} ; E_{\pi}\right)=\frac{\left(x-r_{\pi}\right)}{E_{\pi} x\left(1-r_{\pi}\right)^{2}} \Theta\left(x-r_{\pi}\right)$,

with $x=E_{\mu} / E_{\pi}$ and $r_{\pi}=\left(m_{\mu} / m_{\pi}\right)^{2}$.

The injection function of negative left handed and positive right handed muons is

$$
\begin{aligned}
Q_{\mu_{L}^{-}, \mu_{R}^{+}}^{\prime}\left(E_{\mu}^{\prime}, z\right)= & \int_{E_{\mu}^{\prime}}^{\infty} \mathrm{d} E_{\pi}^{\prime} T_{\pi, \mathrm{d}}^{-1}\left(E_{\pi}^{\prime}\right) \\
& \times\left(N_{\pi^{-}}^{\prime}\left(E_{\pi}^{\prime}, z\right) \frac{\mathrm{d} n_{\pi^{-} \rightarrow \mu_{L}^{-}}}{\mathrm{d} E_{\mu}^{\prime}}\left(E_{\mu}^{\prime} ; E_{\pi}^{\prime}\right)\right. \\
& \left.+N_{\pi^{+}}^{\prime}\left(E_{\pi}^{\prime}, z\right) \frac{\mathrm{d} n_{\pi^{+} \rightarrow \mu_{R}^{-}}}{\mathrm{d} E_{\mu}^{\prime}}\left(E_{\mu}^{\prime} ; E_{\pi}^{\prime}\right)\right)
\end{aligned}
$$

Given that $\mathrm{CP}$ invariance implies that $\mathrm{d} n_{\pi^{-} \rightarrow \mu_{L}^{-}} / \mathrm{d} E_{\mu}^{\prime}=$ $\mathrm{d} n_{\pi^{+} \rightarrow \mu_{R}^{+}} / \mathrm{d} E_{\mu}^{\prime}$, and since the total distribution obtained for all charged pions is $N_{\pi}^{\prime}\left(E_{\pi}^{\prime}, z\right)=N_{\pi^{+}}^{\prime}\left(E_{\pi}^{\prime}, z\right)+N_{\pi^{-}}^{\prime}\left(E_{\pi}^{\prime}, z\right)$, it follows that the injection for left handed muons is

$$
\begin{aligned}
Q_{\mu_{L}^{-}, \mu_{R}^{+}}^{\prime}\left(E_{\mu}^{\prime}, z\right)= & \int_{E_{\mu}^{\prime}}^{\infty} \mathrm{d} E_{\pi}^{\prime} T_{\pi, \mathrm{d}}^{-1}\left(E_{\pi}^{\prime}\right) \\
& \times N_{\pi}^{\prime}\left(E_{\pi}^{\prime}, z\right) \frac{\mathrm{d} n_{\pi^{-} \rightarrow \mu_{L}^{-}}}{\mathrm{d} E_{\mu}^{\prime}}\left(E_{\mu}^{\prime} ; E_{\pi}^{\prime}\right) .
\end{aligned}
$$

And in a similar way, the right handed muons injection is

$$
\begin{aligned}
Q_{\mu_{R}^{-}, \mu_{L}^{+}}^{\prime}\left(E_{\mu}^{\prime}, z\right)= & \int_{E_{\mu}^{\prime}}^{\infty} \mathrm{d} E_{\pi}^{\prime} T_{\pi, \mathrm{d}}^{-1}\left(E_{\pi}^{\prime}\right) \\
& \times N_{\pi}^{\prime}\left(E_{\pi}^{\prime}, z\right) \frac{\mathrm{d} n_{\pi^{-} \rightarrow \mu_{R}^{-}}}{\mathrm{d} E_{\mu}^{\prime}}\left(E_{\mu}^{\prime} ; E_{\pi}^{\prime}\right) .
\end{aligned}
$$

Again, the injection functions of muons given by Eqs. (37), (38) are used to work out the distribution $N_{\mu}^{\prime}\left(E^{\prime}, z\right)$ by solving the transport Eq. (25).

\subsection{Electromagnetic radiation}

The main radiative processes considered in this work are: synchrotron radiation, IC emission, $p p$ and $p \gamma$ interactions. For each emission process we can calculate the injection of photons or radiation emissivity, which is given by the general expression of Eq. (5) and represents the number of photons produced per time unit, per volume unit, per photon energy, and per solid angle. In the observer frame, the corresponding emissivity is given by

$Q_{\gamma}\left(E_{\gamma}, z\right)=D Q_{\gamma}^{\prime}\left(E_{\gamma}^{\prime}, z\right)$

where the Doppler factor is $D=\Gamma_{\mathrm{b}}^{-1}\left(1-\beta_{\mathrm{b}} \cos \theta\right)^{-1}$ and $E_{\gamma}=D E_{\gamma}^{\prime}$.

In the case of synchrotron radiation, the emissivity in the jet frame is

$Q_{\gamma, \mathrm{syn}}^{\prime}\left(E_{\gamma}^{\prime}, z\right)=\frac{\epsilon_{\mathrm{syn}}^{\prime}\left(E_{\mathrm{ph}}^{\prime}, z\right)}{4 \pi E_{\gamma}^{\prime}}$. and the IC emissivity is

$$
\begin{aligned}
Q_{\gamma, \mathrm{IC}}^{\prime}\left(E_{\gamma}^{\prime}, z\right)= & \frac{r_{\mathrm{e}}^{2} c}{2} \int_{E_{\mathrm{ph}}^{\prime(\min )}}^{E_{\gamma}^{\prime}} \mathrm{d} E_{\mathrm{ph}}^{\prime} \frac{n_{\mathrm{ph}}^{\prime}\left(E_{\mathrm{ph}}^{\prime}, z\right)}{E_{\mathrm{ph}}} \\
& \times \int_{E_{\min }^{\prime}}^{E_{\max }^{\prime}} \mathrm{d} E^{\prime} \frac{N_{\mathrm{e}}^{\prime}\left(E^{\prime}, z\right)}{\gamma_{\mathrm{e}}^{2}} F(q),
\end{aligned}
$$

where $r_{\mathrm{e}}$ is the classical electron radius, and we integrate in the target photon energy $E_{\mathrm{ph}}^{\prime}$ and in the electron energies $E^{\prime}$ between

$E_{\text {min }}^{\prime}=\frac{E_{\gamma}^{\prime}}{2}+\frac{m_{\mathrm{e}} c^{2}}{2} \sqrt{\frac{E_{\gamma}^{\prime}}{2 E_{\mathrm{ph}}^{\prime}}+\frac{E_{\gamma}^{\prime 2}}{2 m_{\mathrm{e}}^{2} c^{4}}}$

and

$E_{\max }^{\prime}=\frac{E_{\gamma}^{\prime}}{1-\frac{E_{\gamma}^{\prime}}{E_{\mathrm{ph}}^{\prime}}}$.

With respect to the hadronic contribution to the total emission, the photon emissivity due to $p p$ interactions is obtained as

$$
\begin{aligned}
Q_{\gamma, p p}^{\prime}\left(E_{\gamma}^{\prime}, z\right)= & n_{\mathrm{c}}^{\prime}(z) c \int_{0}^{1} \frac{\mathrm{d} x}{x} N_{\mathrm{p}}^{\prime}\left(\frac{E_{\gamma}^{\prime}}{x}, z\right) \\
& \times F_{\gamma}\left(x, \frac{E_{\gamma}^{\prime}}{x}\right) \sigma_{p p}^{(\text {inel })}\left(\frac{E_{\gamma}^{\prime}}{x}\right),
\end{aligned}
$$

where the function $F_{\gamma}\left(x, E^{\prime}\right)$ is the same as defined by Kelner et al. (2006), for a proton energy $E^{\prime}=E_{\gamma}^{\prime} / x$.

For the $\gamma$-ray emissivity due to $p \gamma$ interactions we use the expression

$$
\begin{aligned}
Q_{\gamma, p \gamma}^{\prime}\left(E_{\gamma}^{\prime}, z\right)= & \frac{1}{4 \pi} \int_{E_{\gamma}^{\prime}} \frac{\mathrm{d} E^{\prime}}{E^{\prime}} N_{\mathrm{p}}^{\prime}\left(E^{\prime}, z\right) \\
& \times \int_{E_{\mathrm{ph}}^{(\min )}}^{E_{\gamma}^{\prime}} \mathrm{d} E_{\mathrm{ph}}^{\prime} n_{\mathrm{ph}}^{\prime}\left(E_{\mathrm{ph}}^{\prime}, z\right) \Phi\left(\eta_{\mathrm{p} \gamma}, x\right),
\end{aligned}
$$

where $E_{\gamma}^{\prime}=x E^{\prime}, \eta_{\mathrm{p} \gamma}=\frac{4 E^{\prime} E_{\mathrm{ph}}^{\prime}}{m_{\mathrm{p}}^{2} c^{4} x}$

$E_{\mathrm{ph}}^{(\mathrm{min})}=\frac{m_{\mathrm{p}}^{2} c^{4} x}{4 E_{\gamma}^{\prime}}\left[2\left(\frac{m_{\pi_{0}}}{m_{\mathrm{p}}}\right)+\left(\frac{m_{\pi_{0}}}{m_{\mathrm{p}}}\right)^{2}\right]$,

and with the function $\Phi\left(\eta_{\mathrm{p} \gamma}, x\right)$ as tabulated in Kelner \& Aharonian (2008).

\subsection{Internal and external photon absorption}

We consider the absorption of the radiation due to photoionization processes at $\mathrm{eV}$ energies, and also due to $e^{-} e^{+}$creation by $\gamma \gamma$ and $\gamma N$ interactions at high energies in the source.

For $\gamma N$ interactions, we take as target the material along the line of sight corresponding to each particular object, which is measured through the column density of neutral hydrogen $N_{\mathrm{H}}$. The absorption cross section $\sigma_{\gamma N}$ in this case is taken as in Ryter (1996) for $E_{\gamma}<1 \mathrm{keV}$, assuming that the medium is composed by atomic hydrogen and dust within galactic abundances (see Fig. 1). This cross section includes the effects of photoionizaton for $E_{\gamma}>13.6 \mathrm{eV}$ and scattering with dust below this energy. In the present work we do not consider recombination, the inverse process of photoionization (see Appendix B). The resulting optical depth for $\gamma N$ interactions is approximated as

$\tau_{\gamma \mathrm{H}}\left(E_{\gamma}\right)=N_{\mathrm{H}} \sigma_{\gamma N}\left(E_{\gamma}\right)$. 
M. M. Reynoso et al.: Lepto-hadronic model for FR I radiogalaxies

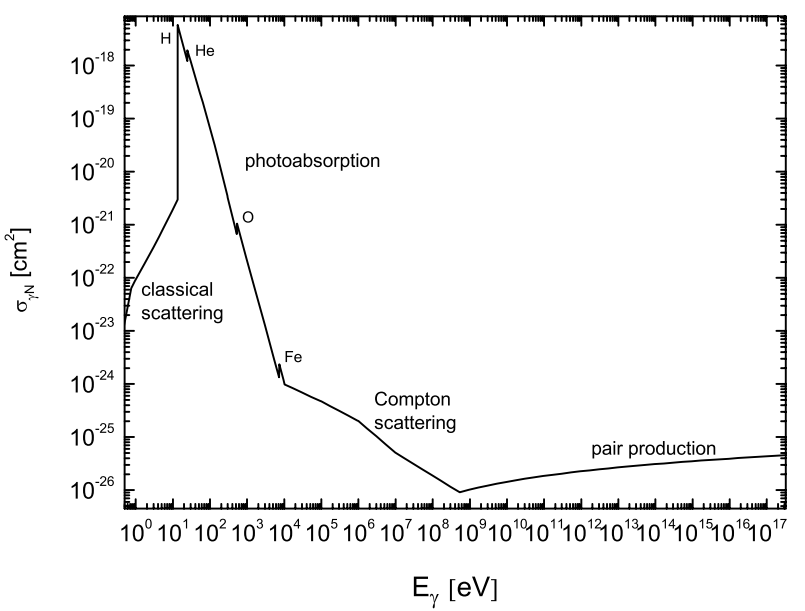

Fig. 1. Cross section for $\gamma N$ interactions. The absorption edges corresponding to the different elements are indicated.

It can be seen from Fig. 1 that besides the large absorption edge corresponding to the ionization energy of hydrogen, three aditional absorption edges are included in the cross section. They correspond to different ionization energies of helium, K-shell electrons of oxygen, and iron. In the present context, as can be seen below, we find no significant features associated with these aditional absorption edges (e.g. Cruddace et al. 1974; Ghisellini et al. 1999). The possible re-emission of lines is beyond the scope of this work, and could be studied, e.g., as in Ghisellini et al. (1999). For energies above $1 \mathrm{keV}$ we take the $\gamma N$ cross section from Amsler et al. (2008), to account for Compton scattering and $e^{ \pm}$production. In Fig. 1 we show the cross section used for $\gamma N$ interactions.

In the case of $\gamma \gamma$ interactions, the main radiation target is considered to be the synchrotron photons inside the jet, which are characterized by a radiation density $n_{\mathrm{ph}}^{\prime}\left(E_{\mathrm{ph}}^{\prime}, z\right)$. If a dissipationless accretion disk is present, no other significant photon field is relevant for gamma-ray absorption. The cross section is given by

$$
\begin{aligned}
\sigma_{\gamma \gamma}\left(E_{\gamma}^{\prime}, E_{\mathrm{ph}}^{\prime}\right)= & \frac{3 \sigma_{\mathrm{T}}}{16}\left(1-\beta_{\mathrm{e}}^{2}\right) \\
& \times\left[2 \beta_{\mathrm{e}}\left(\beta_{\mathrm{e}}^{2}-2\right)+\left(3-\beta_{\mathrm{e}}^{4}\right) \ln \left(\frac{1+\beta_{\mathrm{e}}}{1-\beta_{\mathrm{e}}}\right)\right],
\end{aligned}
$$

with

$\beta_{\mathrm{e}}=\left[1-\frac{2 m_{\mathrm{e}}^{2} c^{4}}{E_{\gamma}^{\prime} E_{\mathrm{ph}}^{\prime}\left(1-\cos \theta_{\gamma \gamma}\right)}\right]^{1 / 2}$,

where $\theta_{\gamma \gamma}$ is the angle of interaction between the incident photons. For $\gamma$-rays produced at a position $z$ in the jet, we assume that they undergo collisions over a length $r_{\mathrm{j}}(z) / \sin \theta^{\prime}$ inside the jet with a target radiation field that is isotropic in the jet frame. The corresponding optical depth is calculated using the general expression of Gould \& Schréder (1967) to obtain

$$
\begin{aligned}
\tau_{\gamma \gamma}^{(\mathrm{int})}\left(E_{\gamma}^{\prime}, z\right)= & 2 \pi \int_{0}^{\frac{r_{\mathrm{j}}(z)}{\sin \theta^{\prime}}} \mathrm{d} l^{\prime} \int_{-1}^{1} \mathrm{~d} x^{\prime}\left(1-x^{\prime}\right) \\
& \times \int_{\frac{2 m_{\mathrm{e}}^{2} c^{4}}{E_{\gamma}^{\left(1-x^{\prime}\right)}}}^{\infty} \mathrm{d} E_{\mathrm{ph}}^{\prime} n_{\mathrm{ph}}^{\prime}\left(E_{\mathrm{ph}}^{\prime}, z^{\prime}\left(l^{\prime}\right)\right) \sigma_{\gamma \gamma}\left(E_{\gamma}^{\prime}, E_{\mathrm{ph}}^{\prime}\right) .
\end{aligned}
$$

In Fig. 2 we show the optical depths obtained for the case of Cen $\mathrm{A}$ and $\mathrm{M} 87, \tau_{\gamma N}$ and $\tau_{\gamma \gamma}$, the latter evaluated within
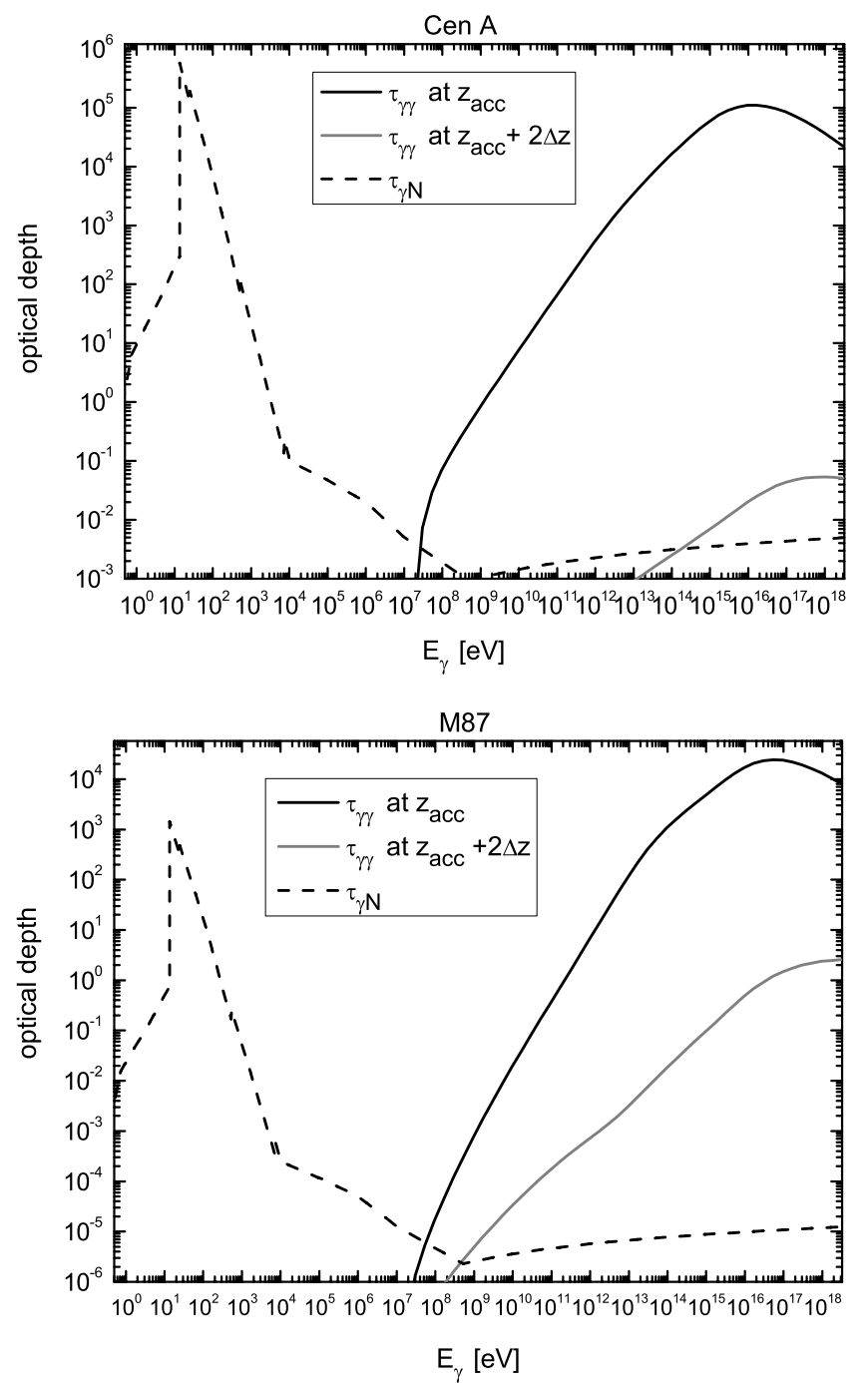

Fig. 2. Optical depth for Cen A and M 87 at different distances form the black hole.

the injection zone and also outside it. The absorbing photons outside the injection zone are those of synchrotron emission of protons and secondary $e^{ \pm}$, which in that case are also included in $n_{\mathrm{ph}}^{\prime}\left(E_{\mathrm{ph}}^{\prime}, z\right)$. It can be seen from this plot that very important absorption occurs at low energies by photoionization, and also at VHE through internal $\gamma \gamma$ interactions. The latter effect takes place mainly at the injection zone, since for $z>z_{\text {acc }}+\Delta z$, the optical depth is much lower.

Taking into account the total optical depth $\tau_{\gamma}\left(E_{\gamma}, z\right)=$ $\tau_{\gamma \gamma}\left(E_{\gamma}, z\right)+\tau_{\gamma N}\left(E_{\gamma}\right)$, the differential photon flux at Earth, i.e., the number of photons with energy $E_{\gamma}$, per unit energy, per unit area, per unit time, can be calculated as

$\frac{\mathrm{d} \Phi_{\gamma}\left(E_{\gamma}\right)}{\mathrm{d} E_{\gamma}}=\frac{1}{d^{2}} \int \mathrm{d} V Q_{\gamma}\left(E_{\gamma}, z\right) \exp \left[-\tau_{\gamma}\left(E_{\gamma}, z\right)\right]$,

where $d$ is the distance from the source to Earth.

\subsection{Neutrino emission}

Neutrinos arise from direct pion decays plus muon decays with a total emissivity

$Q_{v_{\mu}}^{\prime}\left(E^{\prime}, z\right)=Q_{\pi \rightarrow v_{\mu}}^{\prime}\left(E^{\prime}, z\right)+Q_{\mu \rightarrow v_{\mu}}^{\prime}\left(E^{\prime}, z\right)$, 
which correspond to the observer frame and transforms according to Eq. (39). The contribution from pion decays $\left(\pi^{+} \rightarrow \mu^{+} v_{\mu}\right.$, $\pi^{-} \rightarrow \mu^{-} \bar{v}_{\mu}$ ) is represented in the co-moving frame by

$$
\begin{aligned}
Q_{\pi \rightarrow v_{\mu}}^{\prime}\left(E^{\prime}, z\right)= & \int_{E^{\prime}}^{\infty} \mathrm{d} E_{\pi} T_{\pi, \mathrm{d}}^{-1}\left(E_{\pi}\right) N_{\pi}^{\prime}\left(E_{\pi}, z\right) \\
& \times \frac{\Theta\left(1-r_{\pi}-x\right)}{E_{\pi}\left(1-r_{\pi}\right)},
\end{aligned}
$$

with $x=E^{\prime} / E_{\pi}$ and $T_{\pi, \mathrm{d}}=\gamma_{\pi} 2.6 \times 10^{-8} \mathrm{~s}$.

The neutrino emissivity for muon decays $\left(\mu^{-} \rightarrow e^{-} \bar{v}_{\mathrm{e}} v_{\mu}\right.$, $\left.\mu^{+} \rightarrow e^{+} v_{\mathrm{e}} \bar{v}_{\mu}\right)$ can be calculated as

$$
\begin{aligned}
Q_{\mu \rightarrow v_{\mu}}^{\prime}\left(E^{\prime}, z\right)= & \sum_{i=1}^{4} \int_{E^{\prime}}^{\infty} \frac{\mathrm{d} E_{\mu}}{E_{\mu}} T_{\mu, \mathrm{d}}^{-1}\left(E_{\mu}\right) N_{\mu_{i}}^{\prime}\left(E_{\mu}, z\right) \\
& \times\left[\frac{5}{3}-3 x^{2}+\frac{4}{3} x^{3}\right] .
\end{aligned}
$$

In this expression, $x=E^{\prime} / E_{\mu}, \mu_{1,2}=\mu_{L}^{-,+}, T_{\mu, \mathrm{d}}=\gamma_{\mu} 2.2 \times 10^{-6} \mathrm{~s}$, and $\mu_{3,4}=\mu_{R}^{-,+}$(see Lipari et al. 2007). The differential neutrino flux can be computed using a expression analogous to Eq. (50).

The synchrotron cooling of $\mu \mathrm{s}$ and $\pi \mathrm{s}$, which is taken into account in our model, will be traduced on a lower neutrino emissivity as it was previously shown in Reynoso \& Romero (2009).

\subsection{Secondary $e^{+} e^{-}$production}

We consider the production of secondary electrons and positrons in the jet through $p \gamma, p p$, and $\gamma \gamma$ interactions. The inelastic $p \gamma$ collisions with can produce $e^{-} e^{+}$pairs apart from pions as discussed above. We consider this direct production of pairs through the injection function in the jet frame

$$
\begin{aligned}
Q_{\mathrm{e}^{ \pm}, \mathrm{p} \gamma}^{\prime}\left(E^{\prime}, z\right)= & 2 \int_{m_{\mathrm{p}} c^{2}}^{\infty} \mathrm{d} E_{\mathrm{p}}^{\prime} N_{\mathrm{p}}^{\prime}\left(E_{\mathrm{p}}^{\prime}, z\right) \\
& \times \omega_{\mathrm{p} \gamma}^{(e)}\left(E_{\mathrm{p}}^{\prime}, z\right) \delta\left(E^{\prime}-\frac{m_{\mathrm{e}}}{m_{\mathrm{p}}} E_{\mathrm{p}}^{\prime}\right),
\end{aligned}
$$

where the $\omega_{\mathrm{p} \gamma, \mathrm{e}^{ \pm}}\left(E_{\mathrm{p}}^{\prime}\right)$ is the collision frequency given by

$$
\begin{aligned}
\omega_{\mathrm{p} \gamma}^{\prime(e)}\left(E_{\mathrm{p}}^{\prime}, z\right)= & \frac{c}{2 \gamma_{\mathrm{p}}^{2}} \int_{\frac{\epsilon_{\mathrm{th}}}{2 \gamma_{\mathrm{p}}}}^{\infty} \mathrm{d} E_{\mathrm{ph}}^{\prime} \frac{n_{\mathrm{ph}}^{\prime}\left(E_{\mathrm{ph}}^{\prime}, z\right)}{E_{\mathrm{ph}}^{\prime 2}} \\
& \times \int_{\epsilon_{\mathrm{th}}^{(e)}}^{2 \epsilon \gamma_{\mathrm{p}}} \mathrm{d} \epsilon^{\prime} \sigma_{\mathrm{p} \gamma}^{(e)}\left(\epsilon^{\prime}\right) \epsilon^{\prime} .
\end{aligned}
$$

Here, the photopair cross section $\sigma_{\mathrm{p} \gamma}^{(e)}$ is considered as in (Begelman et al. 1990).

As discussed above, the inelastic $p p$ and $p \gamma$ collisions produce pions that decay giving muons. The decay products of muons include electrons and positrons. The decay spectrum of the electrons and positrons are taken to be equal to that of muon neutrinos and anti-neutrinos (see e.g. Gaisser 1990). Thus, the injection of electrons and positrons from muon decay after $p p$ and $p \gamma$ interactions is considered following Schlickeiser (2002).
As for the $e^{+} e^{-}$pairs produced in $\gamma \gamma$ interactions, we consider the injection according to Aharonian et al. (1983),

$$
\begin{aligned}
Q_{\mathrm{e}^{ \pm}, \gamma \gamma}^{\prime}\left(E^{\prime}, z\right)= & \frac{3}{32} \frac{\sigma_{\mathrm{T}} c}{m_{\mathrm{e}} c^{2}} \int_{\gamma_{\mathrm{e}}}^{\infty} \mathrm{d} \epsilon_{\gamma} \int_{\frac{\epsilon_{\gamma}}{4 \gamma_{\mathrm{e}}\left(\epsilon_{\gamma}-\gamma_{\mathrm{e}}\right)}}^{\infty} \mathrm{d} \omega \frac{n_{\gamma}\left(\epsilon_{\gamma}, z\right)}{\epsilon^{3}} \\
& \times \frac{n_{\mathrm{ph}}^{\prime}\left(\omega m_{\mathrm{e}} c^{2}, z\right)}{\omega^{2}}\left\{\frac{4 \epsilon_{\gamma}^{2}}{\gamma_{\mathrm{e}}\left(\epsilon_{\gamma}-\gamma_{\mathrm{e}}\right)} \ln \left[\frac{4 \gamma_{\mathrm{e}} \omega\left(\epsilon_{\gamma}-\gamma_{\mathrm{e}}\right)}{\epsilon_{\gamma}}\right]\right. \\
& \left.-8 \epsilon_{\gamma} \omega+\frac{4 \epsilon_{\gamma}^{3} \omega-2 \epsilon_{\gamma}^{2}}{\gamma_{\mathrm{e}}\left(\epsilon_{\gamma}-\gamma_{\mathrm{e}}\right)}-\left(1-\frac{1}{\epsilon_{\gamma} \omega}\right) \frac{\epsilon_{\gamma}^{4}}{\gamma_{\mathrm{e}}^{2}\left(\epsilon_{\gamma}-\gamma_{\mathrm{e}}\right)^{2}}\right\},
\end{aligned}
$$

where $\gamma_{\mathrm{e}}=E^{\prime} /\left(m_{\mathrm{e}} c^{2}\right), \omega=E_{\mathrm{ph}}^{\prime} /\left(m_{\mathrm{e}} c^{2}\right)$, and $\epsilon=E_{\gamma}^{\prime} /\left(m_{\mathrm{e}} c^{2}\right)$. The soft photon density $n_{\mathrm{ph}}^{\prime}$ refers to the synchrotron radiation of electrons, while the density of higher energy photons from IC, $p p$, and $p \gamma$ interactions, is given by

$n_{\gamma}^{\prime}\left(E_{\gamma}^{\prime}, z\right) \approx 4 \pi \frac{Q_{\gamma, \mathrm{IC}}^{\prime}\left(E_{\gamma}^{\prime}, z\right)+Q_{\gamma, p p}^{\prime}\left(E_{\gamma}^{\prime}, z\right)+Q_{\gamma, p \gamma}^{\prime}\left(E_{\gamma}^{\prime}, z\right)}{c /\left(z \tan \xi_{\mathrm{j}}\right)+t_{\gamma \gamma}^{-1}\left(E_{\gamma}^{\prime}, z\right)}$.

Here, the annihilation rate of gamma-rays due to pair production is given by

$$
\begin{aligned}
t_{\gamma \gamma}^{-1}\left(E_{\gamma}^{\prime}, z\right)= & 2 \pi c \int_{-1}^{1} \mathrm{~d} x^{\prime}\left(1-x^{\prime}\right) \\
& \times \int_{\frac{2 m_{\mathrm{c}}^{2} c^{4}}{E_{\gamma}^{\left(1-x^{\prime}\right)}}}^{\infty} \mathrm{d} E_{\mathrm{ph}}^{\prime} n_{\mathrm{ph}}^{\prime}\left(E_{\mathrm{ph}}^{\prime}, z\right) \sigma_{\gamma \gamma}\left(E_{\gamma}^{\prime}, E_{\mathrm{ph}}^{\prime}\right) .
\end{aligned}
$$

The distribution of secondary electrons and positrons in the jet is found solving the transport equation. This allows us to calculate the synchrotron and IC emission produced by these particles.

\section{Application to Centaurus A}

In this section we present the results for Centaurus A, which were obtained applying our model with the set of parameters listed on Table 1. The cooling rates for high energy electrons and protons for this configuration are shown in Fig. 3, while the obtained electron and proton distributions $N_{\mathrm{e}}^{\prime}\left(E^{\prime}, z\right)$ and $N_{\mathrm{p}}^{\prime}\left(E^{\prime}, z\right)$ are presented in Fig. 4.

We can see in Fig. 3 that the relevant energy looses are due to synchrotron cooling for electrons and $p \gamma$ interactions for protons. Since the plot corresponds to the injection zone $\left(z=z_{\text {acc }}\right)$, $p \gamma$ interactions are favored by a large density of target photons corresponding to the synchrotron emission of electrons. These photohadronic interactions are not so important for protons outside the injection zone.

It is important to notice that the maximum energy achievable for the protons in this context is of $2 \times 10^{7} \mathrm{GeV}$. These protons can not account for the UHE cosmic rays detected by the Pierre Auger Observatory in the direction of Cen A but it gives a hint to other explanations. If neutrons of the same energy are produced by pion photoproduction inside the jet, these could been beamed along the jet and decay into protons near the outer radio lobes, where they would be re-accelerated up to the observed energies by Auger. Other possibilities were proposed by Rieger \& Aharonian (2009), with particles that go through shear acceleration inside the jet, and by Romero et al. (1996), who considered that the UHE cosmic rays are produced at the extended radio lobes. If this is the case, it has been estimated by Hardcastle et al. (2009) that the minimum jet power required is roughly $L_{\mathrm{j}}^{(\mathrm{kin})}>3.5 \times 10^{43} \mathrm{erg} \mathrm{s}^{-1}$, which is consistent with the value assumed here. 
M. M. Reynoso et al.: Lepto-hadronic model for FR I radiogalaxies
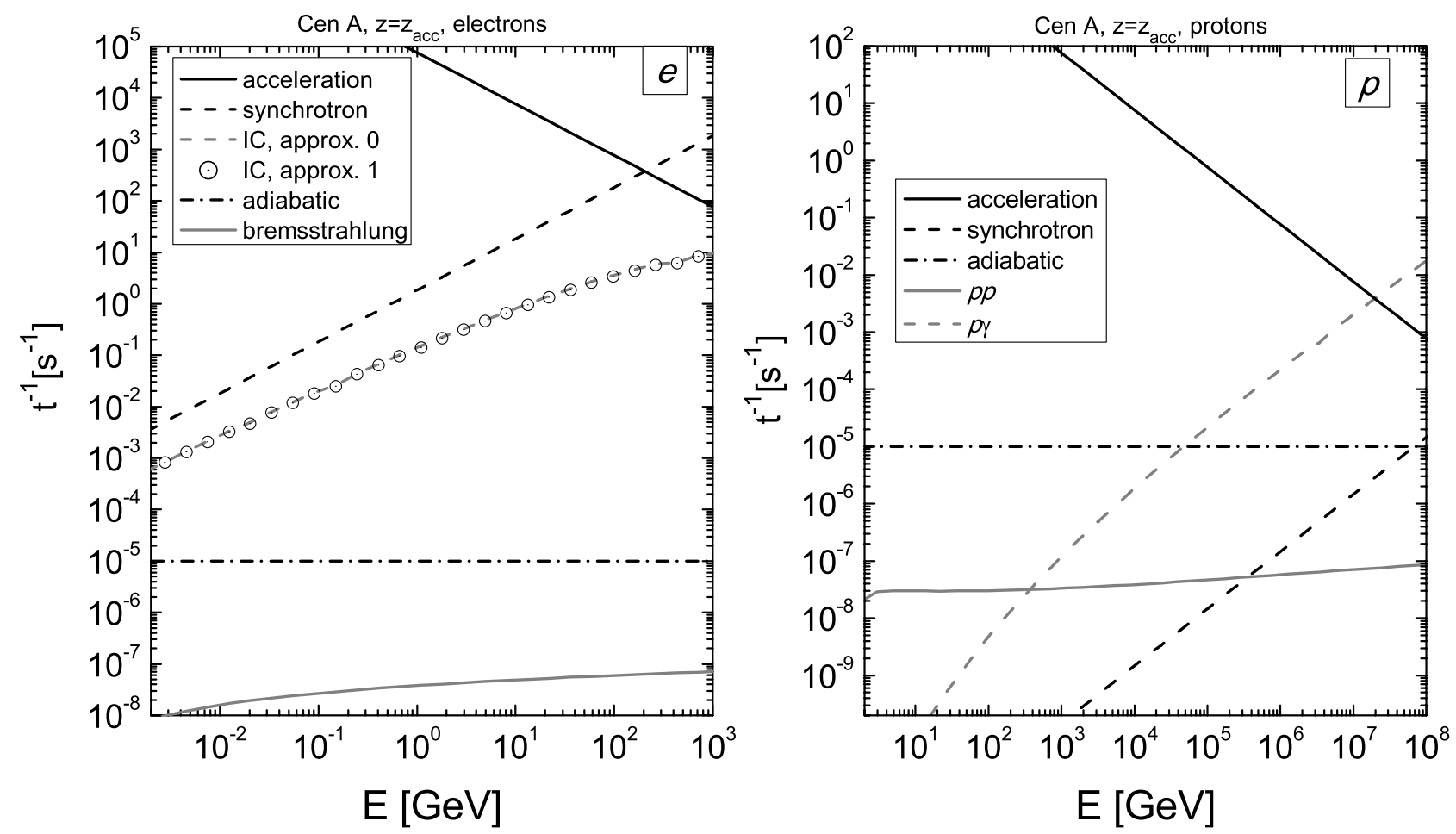

Fig. 3. Accelerating and cooling rates for electrons (left $)$ and for protons (right) at a distance $z=z_{\text {acc }}$ from the central engine of Cen A. In the case of electrons, the light-grey dashed line correspond to the first calculation of the IC cooling rate taken into account only synchrotron cooling to obtain the electron distribution. A second approximation to the electron distribution leads to the IC cooling rate indicated in white circles.

Table 1. Model parameters for Cen A.

\begin{tabular}{|c|c|}
\hline Parameter & Value \\
\hline$M_{\mathrm{bh}}:$ black hole mass & $10^{8} M_{\odot}$ \\
\hline$R_{\mathrm{g}}:$ gravitational radius & $1.47 \times 10^{13} \mathrm{~cm}$ \\
\hline$L_{\mathrm{j}}^{(\mathrm{kin})}$ : jet kinetic power at $z_{0}$ & $6.28 \times 10^{44} \mathrm{erg} \mathrm{s}^{-1}$ \\
\hline$q_{\mathrm{j}}:$ ratio $2 L_{\mathrm{j}}^{(\mathrm{kin})} / L_{\mathrm{Edd}}$ & 0.1 \\
\hline$\Gamma_{\mathrm{b}}\left(z_{0}\right)$ : bulk Lorentz factor of the jet at $z_{0}$ & 3 \\
\hline$\theta$ : viewing angle & $30^{\circ}$ \\
\hline$\xi_{\mathrm{j}}:$ jet's half-opening angle & $2.5^{\circ}$ \\
\hline$q_{\text {rel }}:$ jet's content of relativistic particles & 0.05 \\
\hline$a$ : hadron-to-lepton power ratio & 0.025 \\
\hline$z_{0}:$ jet's launching point & $50 R_{\mathrm{g}}$ \\
\hline$q_{\mathrm{m}}$ : magnetic to kinetic energy ratio at $z_{\mathrm{acc}}$ & 0.38 \\
\hline$z_{\text {acc }}$ : injection point & $132 R_{\mathrm{g}}$ \\
\hline$\Delta z:$ size of injection zone & $2 z_{\mathrm{acc}} \tan \xi_{\mathrm{j}}=11.5 R_{\mathrm{g}}$ \\
\hline$B\left(z_{\text {acc }}\right)$ : magnetic field at $z_{\text {acc }}$ & $3065 \mathrm{G}$ \\
\hline$m$ : index for magnetic field dependence on $z$ & 1.5 \\
\hline$s$ : injection spectral index & 1.8 \\
\hline$\eta:$ acceleration efficiency & $10^{-2}$ \\
\hline$E_{\mathrm{p}}^{(\mathrm{min})}:$ minimum proton energy & $3 \mathrm{GeV}$ \\
\hline$E_{\mathrm{e}}^{(\min )}:$ minimum electron energy & $0.1 \mathrm{GeV}$ \\
\hline$E_{\mathrm{p}}^{(\max )}:$ maximum primary proton energy & $2 \times 10^{7} \mathrm{GeV}$ \\
\hline$E_{\mathrm{e}}^{(\max )}:$ maximum primary electron energy & $200 \mathrm{GeV}$ \\
\hline$N_{\mathrm{H}}:$ column dust density & $10^{23} \mathrm{~cm}^{-2}$ \\
\hline$n_{\mathrm{c}}\left(z_{\mathrm{acc}}\right)$ : cold matter density inside the jet at $z_{\mathrm{acc}}$ & $3 \times 10^{8} \mathrm{~cm}^{-3}$ \\
\hline
\end{tabular}

Figure 4 shows that the electron distribution drops quickly with $z$ at the end of the injection zone, while the proton distribution is also important further along the jet. This is a consequence of the different energy loss rates of the particles: electrons cool very rapidly emitting synchrotron radiation, and protons lose energy at a much lower rate as they propagate along the jet.

The SED of Cen A (see Fig. 5) includes the HESS spectrum in the VHE range together with data from CGRO/COMPTEL (Steinle et al. 1998), RXTE and INTEGRAL (Rothschild et al. 2006). We also include data from HST/NICMOS and WFPC2 (Marconi et al. 2000), SCUBA at $800 \mu \mathrm{m}$ from (Hawarden et al. 1993), ISO and SCUBA (450 $\mu \mathrm{m}$ and $850 \mu \mathrm{m})$ (Mirabel et al. 1999), XMM-Newton, Chandra spectra and the Suzaku data presented in (Abdo et al. 2010). The Fermi/LAT correspond to Cen A core as given in (Abdo et al. 2010), and, finally, we also show the old data from the NASA Extragalactic Database.

The Fermi/LAT analysis revealed that the high-energy spectrum is non-variable over the first ten months of scientific operation of the instrument. This steady behavior is also supported by the HESS experiment, which also reported a constant flux from Cen A even though CGRO data shows some variability with at least two emission states during the period 1991-2000.

Since the set of measurements composing the SED of Cen A is quite inhomogeneous in time and angular resolution, so one should be very careful in attempting to interpret any fit of all the spectrum simultaneously. In particular, the data that define the bump in the hard-X-rays $(\sim 0.1 \mathrm{MeV})$ have been taken more than 10 years ago with a poor angular resolution and long integration times. The lack of a good spatial resolution makes impossible to distinguish the emission components (jet, nucleus or other radiation sources). Furthermore, we note a discrepancy between the flux normalization of Fermi/LAT and HESS, which is not yet fully understood.

Nevertheless, we have obtained using our model a spectral energy distribution which is basically consistent with the multi wavelength emission from Cen A. 

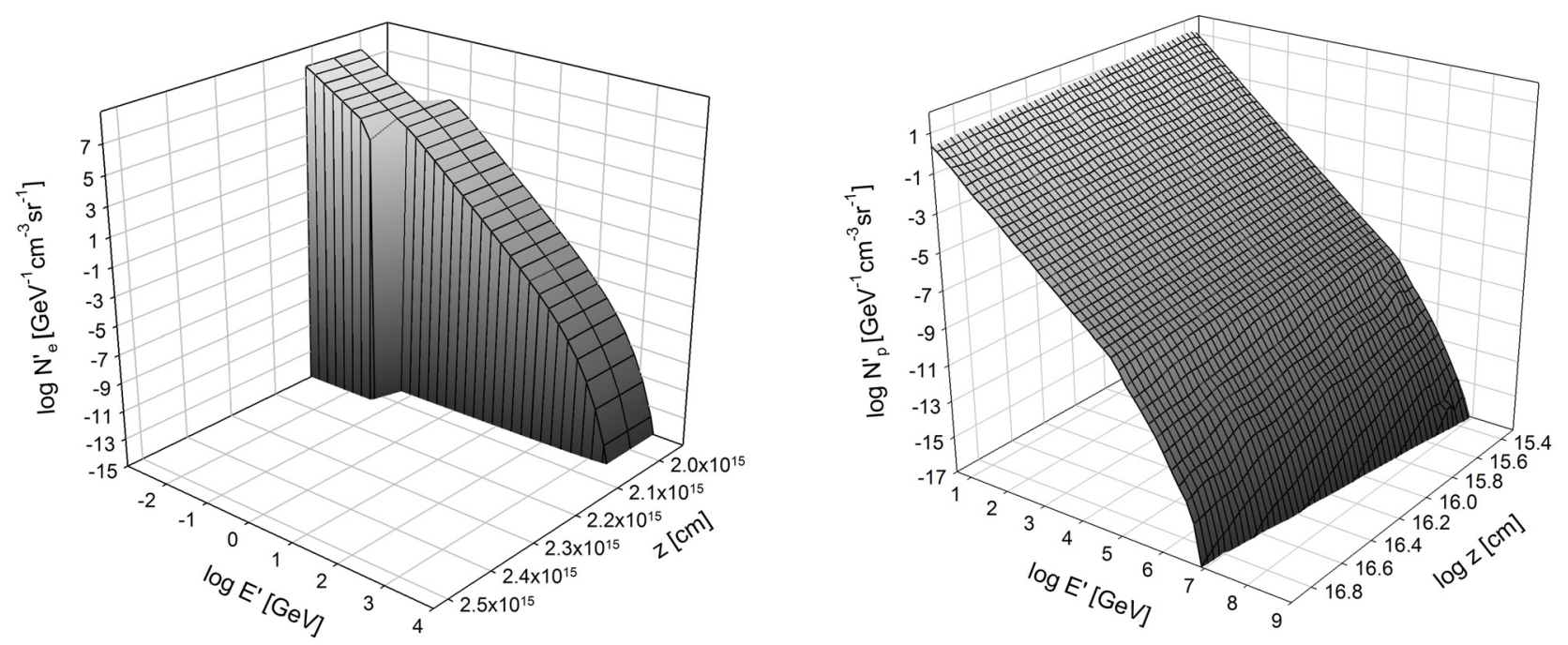

Fig. 4. Distributions of primary particles as function of the energy and the distance to the core inside the Cen A jet. Left: electrons; right: protons.

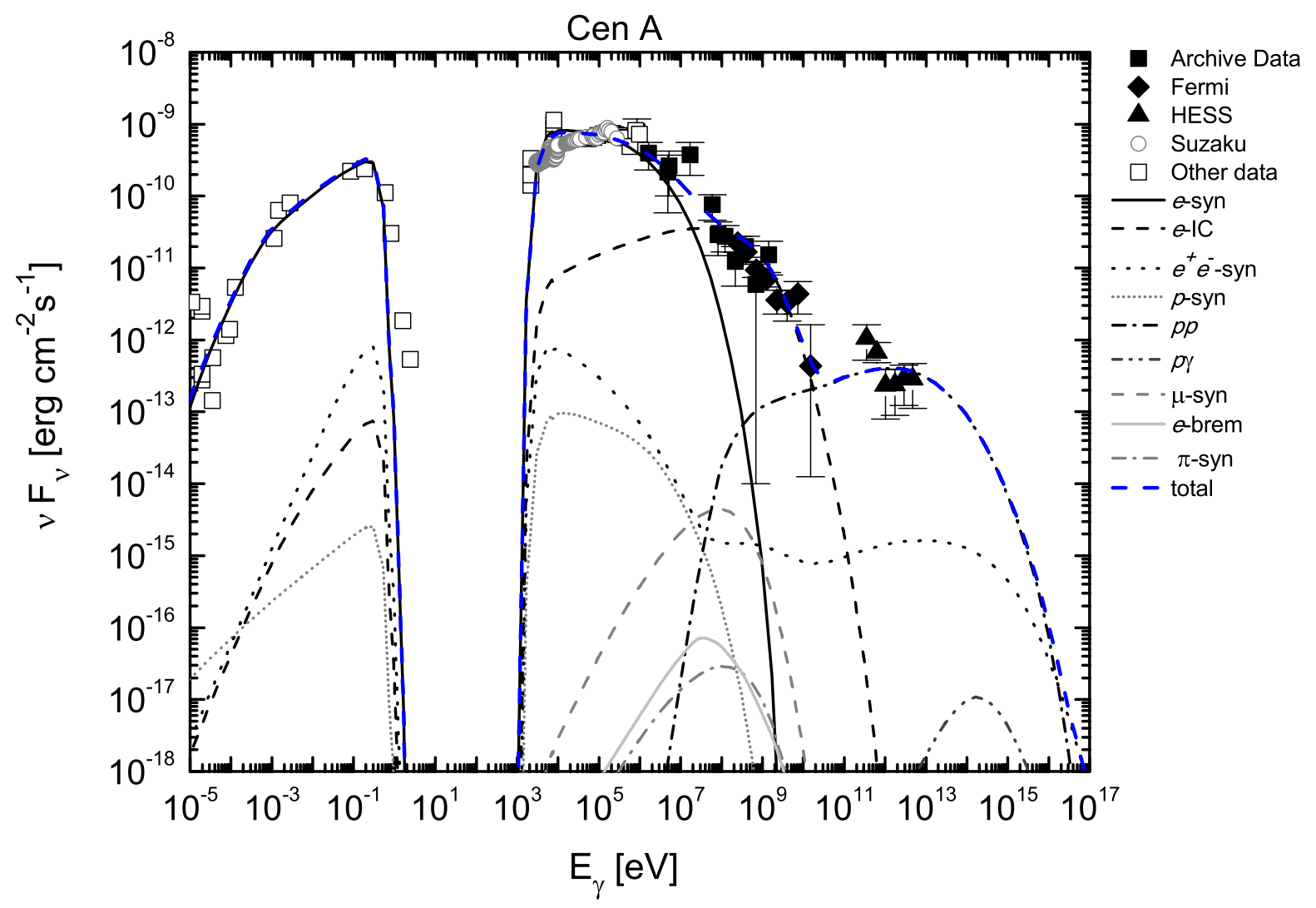

Fig. 5. Model output for the SED of Cen A. The different emission processes are indicated, together with the total output. The recent observational data is also included: Fermi/LAT (black filled diamons) (Abdo et al. 2010) and HESS spectra (black filled triangles). The rest of the data points correspond to the references given in the text.

An important role is played by absorption due to photoionization interactions in the surrounding dust. This arises due to the large value adopted for the column density of neutral hydrogen, $N_{\mathrm{H}}=10^{23} \mathrm{~cm}^{-2}$ (see, e.g. Evans et al. 2004; Morganti et al. 2008). A drastic modulation is then imprinted in the electron synchrotron spectrum, which is responsible for the whole emission in the broadband range $10^{-5}-10^{7} \mathrm{eV}$. Such situation is possible if electrons can be efficiently accelerated $(\eta=0.01)$ to high energies with a rather flat spectral index $(s=1.8)$. The internal $\gamma \gamma$ absorption does not modify significantly the $p p$ contribution of gamma-rays, since it is only important within the injection zone, which is a negligible region of the jet compared to the one in which $p p$ collisions occur (see Fig. 4).

We evaluate the accompanying neutrino output using the same set of parameters. The obtained differential flux, weighted by the squared energy is plotted in Fig. 6 together with the estimated sensitivity of KM3Net for one year of operation (Katz et al. 2009). We can see in this figure that the neutrino signal produced by $p p$ and $p \gamma$ interactions would not be observable by $\mathrm{KM} 3 \mathrm{NeT}$ detector in just one year of observation. It is to be noticed, however, that the sensitivity level shown in the plot actually corresponds to a neutrino spectrum with an $E_{v}^{-2}$ dependence, 
M. M. Reynoso et al.: Lepto-hadronic model for FR I radiogalaxies

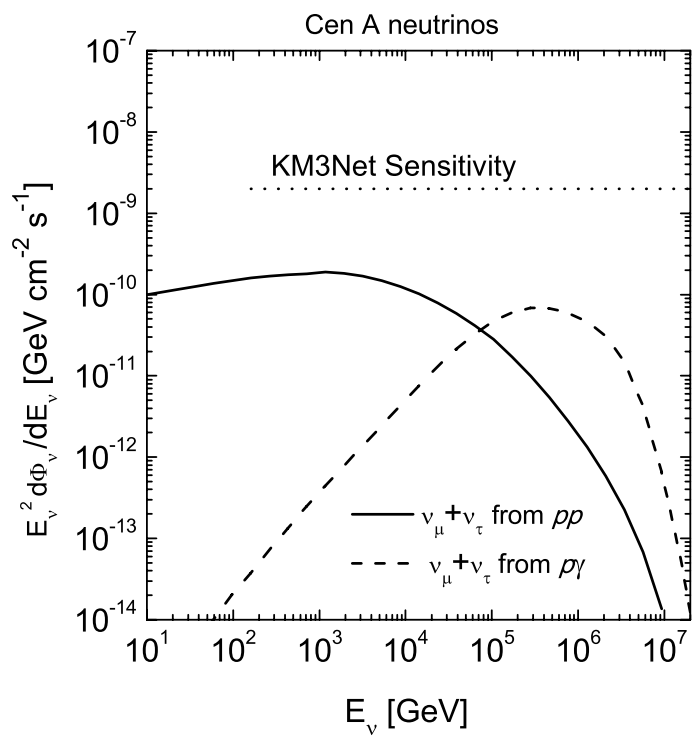

Fig. 6. Differential neutrino flux weighted by the squared energy as predicted by the model for Cen A. Dotted line: approximate KM3Net sensitivity for 1 year operation.

which is a bit steeper than our case. Hence, we can expect that the actual sensitivity for our flux will be better than what is shown here, so with two or three years of data taking it would be possible to achieve detection.

\section{Application to $\mathbf{M} 87$}

In the case of M 87, we have used the set of parameters listed on Table 2 as well as the multi wavelength data available. We show the cooling rates for high energy electrons and protons in Fig. 7, and the electron and proton distributions $N_{\mathrm{e}}^{\prime}\left(E^{\prime}, z\right)$ and $N_{\mathrm{p}}^{\prime}\left(E^{\prime}, z\right)$ in Fig. 8.

We can see in Fig. 7 that the relevant energy looses are due to synchrotron cooling of electrons and protons. Protons also cool significantly through adiabatic losses, and although $p p$ interactions are not dominant, they can give an important radiative output. From the balance between this radiative loss rate and the acceleration rate, the maximum energy achievable for the protons inside the jet is about $9 \times 10^{6} \mathrm{GeV}$. These protons do not escape from the source. The only possibility to obtain a detectable cosmic rays flux at the earth coming from M 87 should depend on neutrons produced by pion photoproduction processes and relativistically beamed along the jet direction and Doppler boosted in energy.

As in the case of Cen A, Fig. 8 shows that electrons radiate only at the base of the jet loosing all their energy rapidly while protons survive longer inside the jet, emmiting enough radiation to account for the VHE $\gamma$ observed emission.

In Fig. 9 we plot the contributions to the SED of M 87 obtained with the different emitting proceeses in our model. We also show the corresponding multi wavelength observational data. The HESS spectrum included in the VHE range corresponds to a low state from the 2004 observing season (Aharonian et al. 2006). Data from Fermi/LAT (Abdo et al. 2009c), 2009 MOJAVE VLBA $15 \mathrm{GHz}$ (Kellermann et al. 2004) and Chandra $\mathrm{X}$-ray measurements of the core (Harris et al. 2009) are also shown. We also include the $3 \sigma$ upper limits of the integrated emission in three hard X-ray bands based on the Swift/BAT dataset (Ajello et al. 2009). Historical measurements of the core are also plotted: VLA 1.5, 5, $15 \mathrm{GHz}$ (Biretta et al. 1991),
Table 2. Model parameters for M 87.

\begin{tabular}{|c|c|}
\hline Parameter & Value \\
\hline$M_{\mathrm{bh}}:$ black hole mass & $6 \times 10^{9} M_{\odot}$ \\
\hline$R_{\mathrm{g}}$ : gravitational radius & $8.87 \times 10^{14} \mathrm{~cm}$ \\
\hline$L_{\mathrm{j}}^{(\mathrm{kin})}:$ jet power at $z_{0}$ & $1.9 \times 10^{46} \mathrm{erg} \mathrm{s}^{-1}$ \\
\hline$q_{\mathrm{j}}:$ ratio $2 L_{\mathrm{j}}^{(\mathrm{kin})} / L_{\mathrm{Edd}}$ & 0.05 \\
\hline$\Gamma_{\mathrm{b}}\left(z_{0}\right)$ : bulk Lorentz factor of the jet at $z_{0}$ & 4 \\
\hline$\theta$ : viewing angle & $30^{\circ}$ \\
\hline$\xi_{\mathrm{j}}:$ jet's half-opening angle & $1.5^{\circ}$ \\
\hline$q_{\text {rel }}:$ jet's content of relativistic particles & 0.05 \\
\hline$a$ : hadron-to-lepton power ratio & 40 \\
\hline$q_{\mathrm{m}}:$ magnetic to kinetic energy ratio at $z_{\text {acc }}$ & 0.35 \\
\hline$z_{0}:$ jet's launching point & $50 R_{\mathrm{g}}$ \\
\hline$z_{\text {acc }}:$ injection point & $143 R_{\mathrm{g}}$ \\
\hline$\Delta z:$ size of injection zone & $z_{\mathrm{acc}} \tan \xi_{\mathrm{j}} / 4=11.5 R_{\mathrm{g}}$ \\
\hline$B\left(z_{\mathrm{acc}}\right)$ : magnetic field at $z_{\mathrm{acc}}$ & $407 \mathrm{G}$ \\
\hline$m$ : index for magnetic field dependence on $z$ & 1.5 \\
\hline$s:$ injection spectral index & 2.4 \\
\hline$\eta:$ acceleration efficiency & $1.5 \times 10^{-5}$ \\
\hline$E_{\mathrm{p}}^{(\mathrm{min})}:$ minimum proton energy & $3 \mathrm{GeV}$ \\
\hline$E_{\mathrm{e}}^{(\mathrm{min})}:$ minimum electron energy & $65 \mathrm{MeV}$ \\
\hline$E_{\mathrm{p}}^{(\max )}:$ maximum primary proton energy & $9 \times 10^{6} \mathrm{GeV}$ \\
\hline$E_{\mathrm{e}}^{(\max )}:$ maximum primary electron energy & $25 \mathrm{GeV}$ \\
\hline$N_{\mathrm{H}}:$ column dust density & $2 \times 10^{20} \mathrm{~cm}^{-2}$ \\
\hline$n_{\mathrm{c}}\left(z_{\mathrm{acc}}\right)$ : cold matter density inside at $z_{\text {acc }}$ & $3.9 \times 10^{6} \mathrm{~cm}^{-3}$ \\
\hline
\end{tabular}

IRAM $89 \mathrm{GHz}$ (Despringre et al. 1996), SMA $230 \mathrm{GHz}$ (Tan et al. 2008), Spitzer 70, $24 \mathrm{~m}$ (Shi et al. 2007), Gemini $10.8 \mathrm{~m}$ (Perlman et al. 2001) and HST optical/UV (Sparks et al. 1996).

We have obtained, using our model, a spectral energy distribution which is basically consistent with the multi wavelength emission from M 87. The spectrum detected by the Fermi/LAT collaboration connects smoothly with the low-state TeV spectrum detected by HESS. Our model reproduces this connection effectively with the $\gamma$-ray emission produced by $p p$ collisions. Contrary to the case of Cen A, this radiative output for M 87 is possible if particles are not so efficiently accelerated $\left(\eta=1.5 \times 10^{-5}\right)$ to high energies with a rather steep spectral index $(s=2.4)$.

The flaring state, which is not shown in Fig. 9, can still be reproduced in our model by $\gamma$-rays from $p p$ interactions if we assume a flatter spectrum of injected protons and a negligible power in relativistic electrons.

The accompanying high energy neutrino flux was calculated using also the set of parameters of Table 2. We show the differential neutrino flux weighted by the squared energy as a function of energy in Fig. 10, where we also plot the estimated sensitivity of IceCube for one year of operation (Ahrens et al. 2004).

As can be seen been from Fig. 10, the neutrino signal associated to M 87 would be hard to detect. However, the sensitivity shown corresponds to a neutrino spectrum with an $E_{v}^{-2}$ dependence, i.e., not so steep as our predicted one. Hence, a more detailed study could help to assess the real detectability of this type of signal (Neronov \& Ribordy 2009b,a).

\section{Conclusions}

We have presented here a lepto-hadronic model describing the particle propagation and interactions in the inclined jets of 

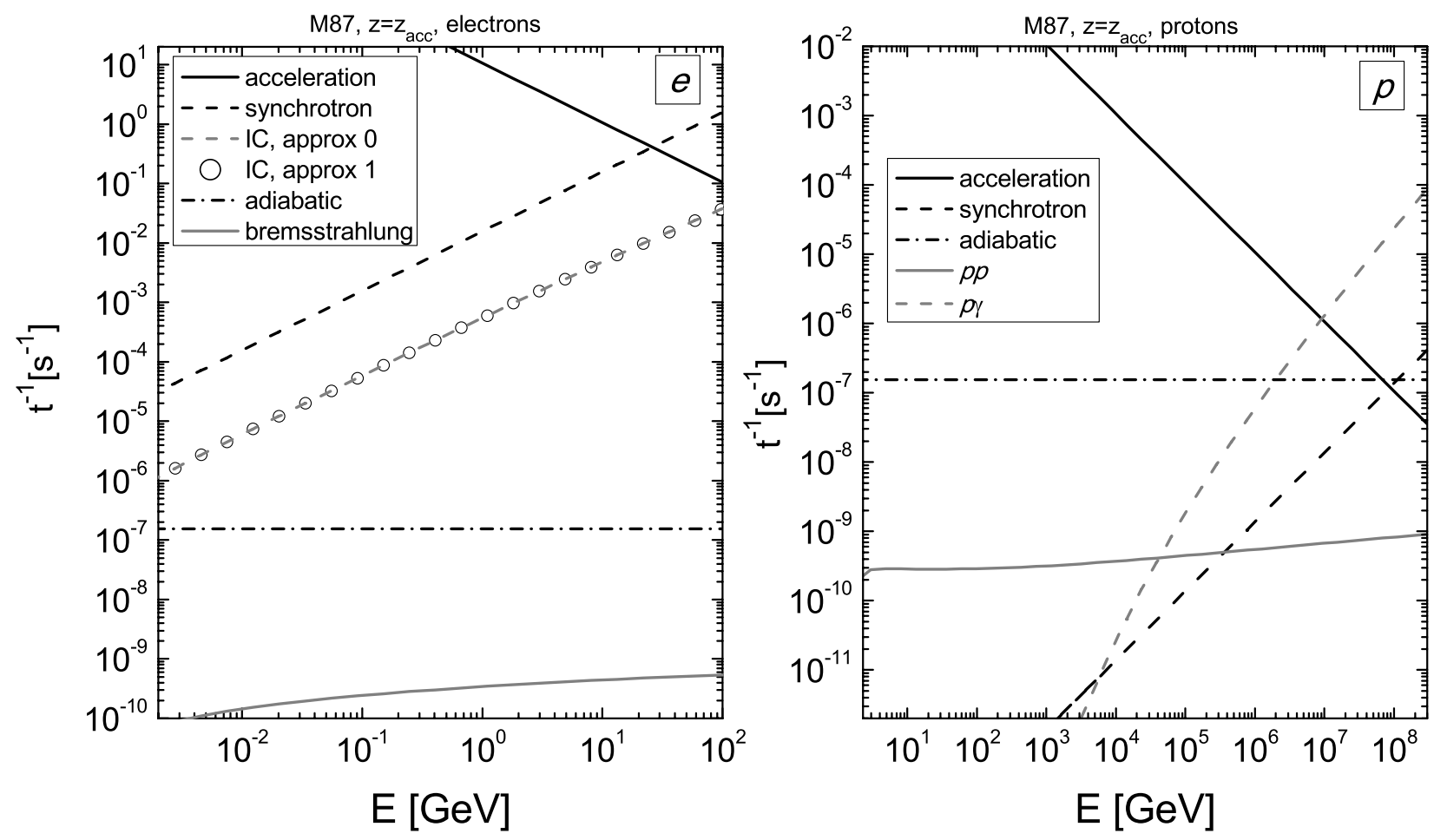

Fig. 7. Accelerating and cooling rates for electrons (left) and for protrons (right) at a distance $z=z_{\text {acc }}$ from the central engine of $\mathrm{M} 87$.
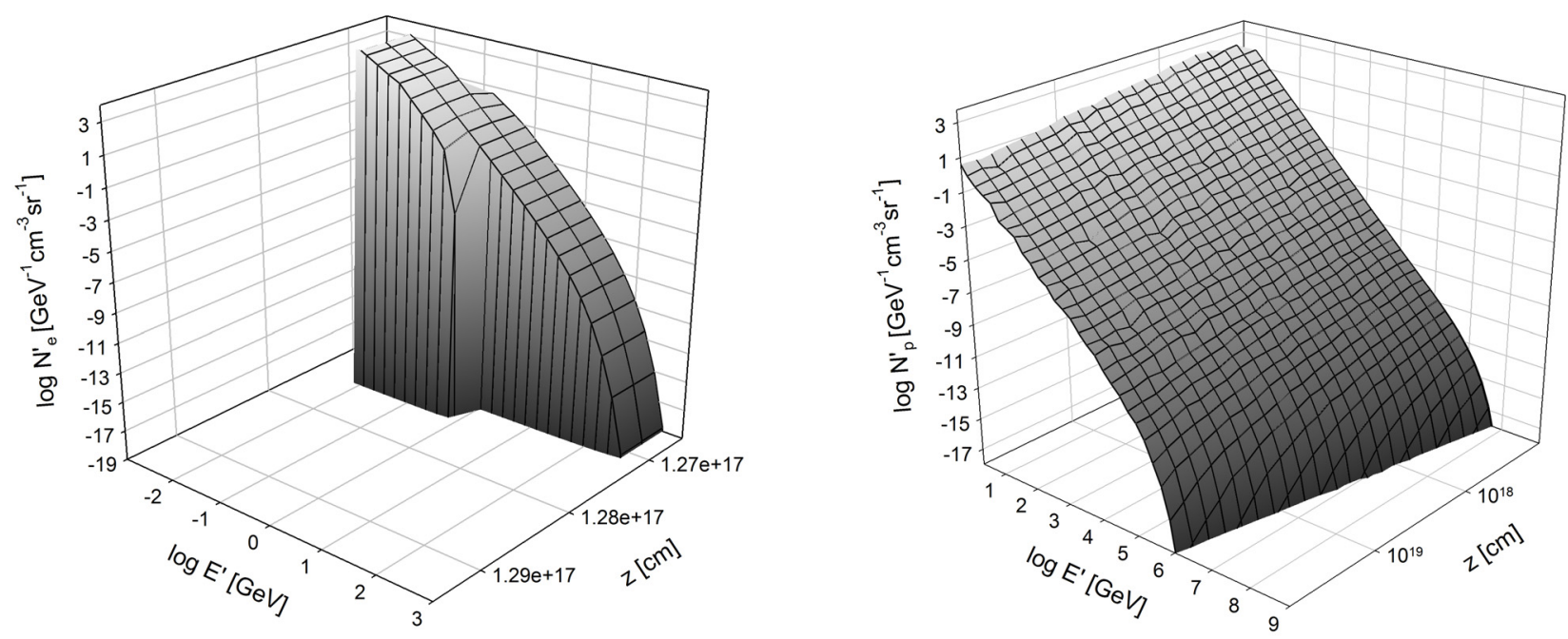

Fig. 8. Distributions of primary particles as function of the energy and the distance to the core inside the M 87 jet. Left: electrons; right: protons.

radiogalaxies. The scenario includes an acceleration zone placed near the jet base, where relativistic particles are injected. The location of this injection zone is fixed in the jet at a distance $z_{\text {acc }}$ from the black hole by requiring that the magnetic energy density is less prominent than the bulk kinetic density. This favors shock formation.

One very important aspect of the present model is that it does not consist of a one-zone treatment, since particles are allowed to convect along the jet, away from the injection zone. To obtain the particle distributions, we have used a stationary one-dimensional transport equation that accounts for energy losses, escape, and convection of particles. The resulting distribution of primary electrons is important mainly in the injection zone, since they undergo a rapid synchrotron cooling. Instead, protons are convected away along the jet, loosing energy gradually (mainly by adiabatic cooling, synchrotron radiation, and $p p$ interactions).
Also they undergo significant $p \gamma$ collisions with the electronsynchrotron photons at the injection zone. The secondary pions and muons produced in the hadronic interactions are also considered, and their distributions are found using the mentioned type of transport equation. This allows to account for the synchrotron cooling suffered before the decay of this secondary, transient particles.

We have found that the radiative output obtained can account for much of the observational data for the two radiogalaxies M 87 and Cen A. As seen in Figs. 5 and 9, the model allows for the possibility of explaining the VHE emission by means of $p p$ interactions. The hard X-ray data corresponds to electron synchrotron radiation whereas the soft- $\gamma$ emission is well reproduced by proton synchrotron and IC emission. Previous hadronic models (e.g. the synchrotron proton blazar of Mücke et al. 2003) could also fit the Fermi energy range for M 87 and the hard 


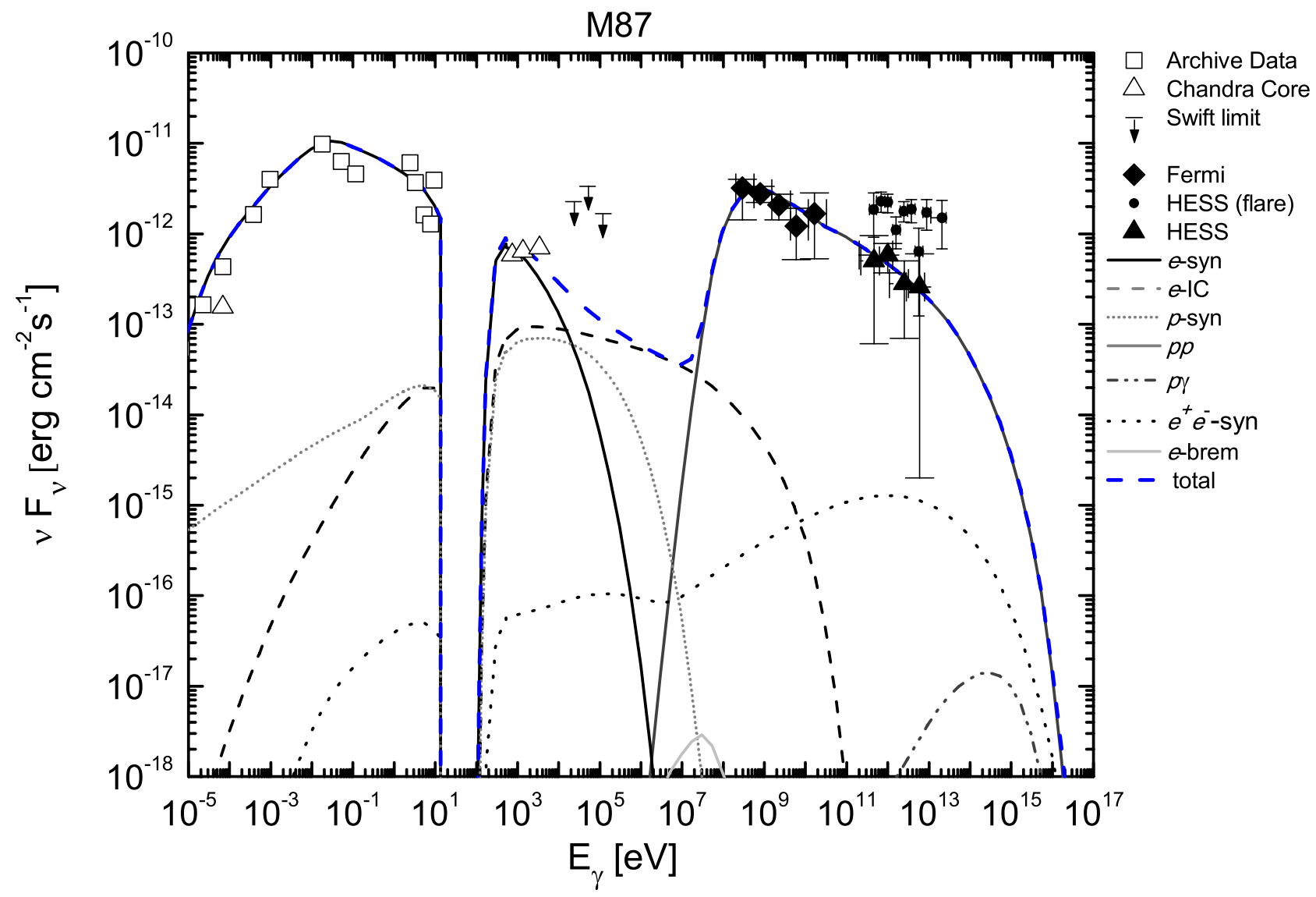

Fig. 9. M 87 SED taking into account the recent Fermi/LAT, HESS spectra in two different states and historical multi wavelength data recompiled in Abdo et al. (2009c). Model output for different processes and particles and the total output are also shown. The model fits the quiescent state.

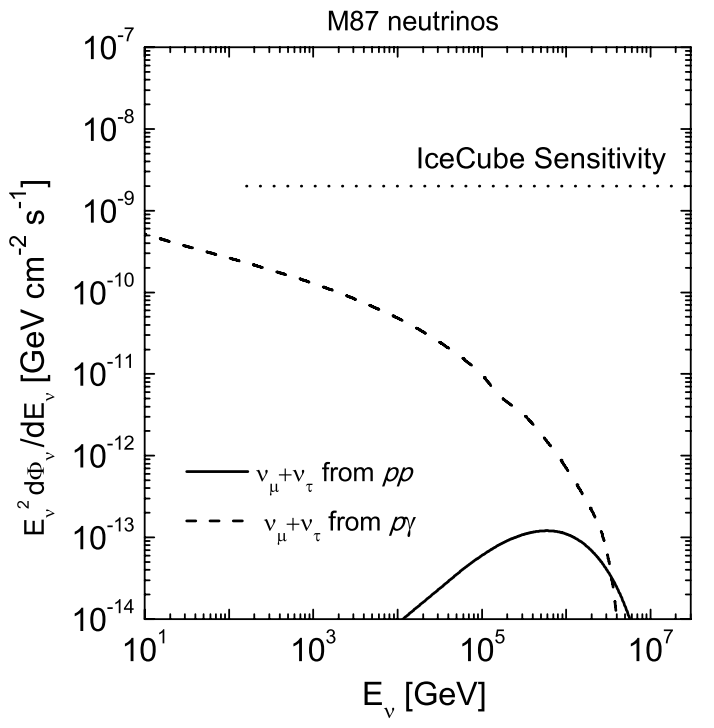

Fig. 10. Differential neutrino flux weighted by the squared energy as predicted by the model for M 87. Dotted line: approximate IceCube sensitivity for 1 year operation.

spectrum measured by HESS (consistent with VERITAS and MAGIC measurements), according to what it is mentioned in Abdo et al. (2009c). A previous one-zone lepto-hadronic model for Cen A was proposed by Orellana \& Romero (2009) just before HESS and Fermi/LAT data was available.
In the present work, we have included the effects of absorption due to photoionization interactions in the surrounding medium. This causes a drastic modulation in the electron synchrotron spectrum, especially for Cen A, which is responsible for the whole emission in the broadband range $\sim 10^{-5}-10^{7} \mathrm{eV}$. In the case of M 87, this type of absorption has a minor impact, since the column density is much less $\left(N_{\mathrm{H}}=2 \times 10^{20} \mathrm{~cm}^{-2}\right.$, Lieu et al. 1996). The internal absorption of gamma-rays is also taken into account, being important only within the injection zone, where the synchrotron emission of primary electrons provide an important absorbing target.

It is important to note that the same model can be used to describe different types of jet. In terms of the broadband photon emission, M 87 data shows a similar luminosity level for high and the low energy ranges. Since the VHE emission is basically determined by $p p$ interactions, we need a large power to be injected in relativistic protons as compared to that injected in electrons. This is because electrons cool completely, mainly by synchrotron emission, and protons undergo an important adiabatic cooling. Hence, in the case of M 87, we need a high value for the proton-to-electron power ratio: $a=40$. A different situation arises for Cen $\mathrm{A}$, where the highest luminosities correspond to energies $E_{\gamma}<10^{7} \mathrm{eV}$, and the HE and VHE luminosities are comparatively lower. In this case, we need more power to be injected in electrons than in protons $\left(a=2.5 \times 10^{-2}\right)$ to allow the broadband electron-synchrotron spectrum to approximately account for all the data for $E_{\gamma}<10^{7} \mathrm{eV}$. Then, for Cen A a lower luminosity in protons is enough to reach the level of the observed $\mathrm{HE}$ and VHE radiation through proton-synchrotron and $p p$ interactions, respectively. A further difference between Cen A and 
M 87 is in the slope of the injected particle distributions: for Cen A, we have a quite flat injection $(s=1.8)$, while for M 87 we find a steeper injection $(s=2.4)$. Also the acceleration efficiency is greater for Cen A than for M 87. This also has an impact in the neutrino spectrum produced, which for $E_{v}>1 \mathrm{TeV}$ appears more difficult to be detected for M 87 than for Cen A.

Finally, we remark that a possible improvement of the present treatment, which is left for future work, should be the inclusion of a time-dependent injection to account for flaring states.

Acknowledgements. This work had the support from the GdR-PCHE, the Observatory of Paris, and CONICET (PIP 112-200801-00587, and PIP 112200901-00078). The authors would like to thank Andreas Zech, Jean-Philippe Lenain, Catherine Boisson and Helene Sol for the very fruitful discussions and the interesting contributions to this work, especially for Cen A. We also thank Gabriela Vila and Chloé Guennou for useful discussions on M 87 and jet physics.

\section{Appendix A: Bulk Lorentz factor evolution}

The sum of the kinetic plus magnetic energy in the jet is

$\mathrm{d} z \pi z^{2} \chi^{2}\left[\rho_{\mathrm{k}}(z)+\rho_{\mathrm{m}}(z)\right]=2 \rho_{\mathrm{m}}\left(z_{0}\right) \mathrm{d} z \pi z_{0}^{2} \chi^{2}$

where $\chi=\tan \xi_{\mathrm{j}}$. Then,

$\rho_{\mathrm{k}}(z)=\frac{B_{0}^{2}}{8 \pi}\left(\frac{z_{0}}{z}\right)^{2}\left[2-\left(\frac{z_{0}}{z}\right)^{2 m-2}\right]$.

Since

$\rho_{\mathrm{k}}(z)=\left[\Gamma_{\mathrm{b}}(z)-1\right] \frac{\dot{m}_{\mathrm{j}} c^{2}}{v_{\mathrm{b}}(z) z^{2} \tan ^{2} \xi_{\mathrm{j}}}$

and given that

$v_{\mathrm{b}}=c \sqrt{1-\frac{1}{\Gamma_{\mathrm{b}}^{2}(z)}}$,

we obtain

$\frac{\Gamma_{\mathrm{b}}\left(\Gamma_{\mathrm{b}}-1\right)}{\sqrt{\Gamma_{\mathrm{b}}^{2}-1}}=\frac{B_{0}^{2} \chi^{2} z_{0}^{2}}{8 \dot{m}_{\mathrm{j}} c}\left[2-\left(\frac{z_{0}}{z}\right)^{2 m-2}\right] \equiv \sqrt{A_{z}}$.

Squaring, we obtain the equation

$\frac{\Gamma_{\mathrm{b}}^{2}\left(\Gamma_{\mathrm{b}}-1\right)}{\Gamma_{\mathrm{b}}+1}=A_{z}$

which has the analytic solution

$$
\begin{aligned}
\Gamma_{\mathrm{b}}(z)= & \frac{1}{3}\left[18 A_{z}+3 \sqrt{3} \sqrt{11 A_{z}^{2}+A_{z}-A_{z}^{3}}+1\right]^{1 / 3}+\frac{1}{3} \\
& +\frac{3 A_{z}+1}{3\left[18 A_{z}+3 \sqrt{3} \sqrt{11 A_{z}^{2}+A_{z}-A_{z}^{3}}+1\right]^{1 / 3}} .
\end{aligned}
$$

For Cen A and M 87 we obtain the results shown in Fig. A.1.

\section{Appendix B: Absorption by photoionization in the surrounding medium}

Here we discuss on the absorption of photons once they espape from the jet and propagate in the ambient medium filled with hydrogen gas. In principle, photons with energies above the
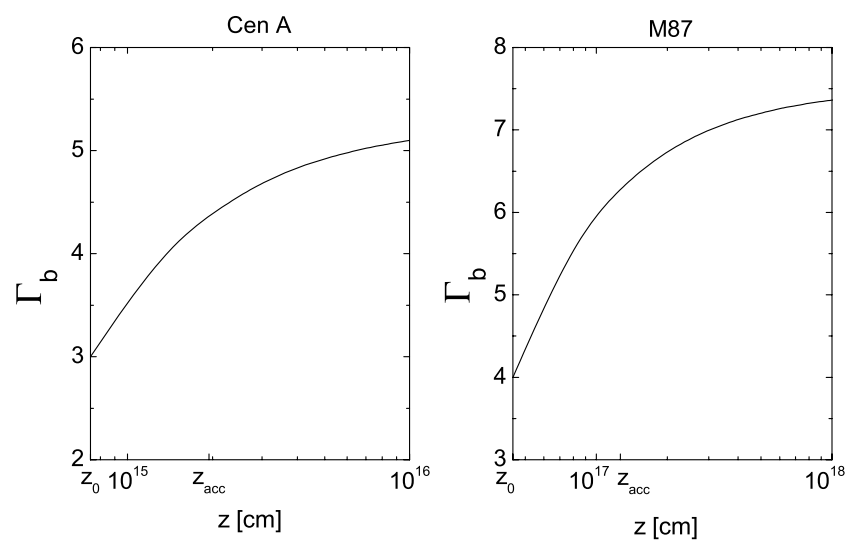

Fig. A.1. Bulk lorentz factor of the jet as a function of the distance to the black hole obtained for Cen A (left panel) and M 87 (right panel).

Lyman edge, $13.6 \mathrm{eV}$, can ionize the neutral hydrogen atoms, and the recombination of electrons and protons can also occur. It is expected that the vicinity of the jet will be highly ionized, but since the emitted radiation can not ionize an infinite volume of gas, then the gas must become neutral at some point (see e.g. Spitzer 1978).

A crude estimation of the size of the ionized zone can be attempted assuming a spherical symmetry of the emitting zone and of the radiation emitted. Considering that the gas extends upto a distance $R_{\mathrm{H}}$ from the emitting zone, we adopt an uniform density of hydrogen $n_{\mathrm{H}}=N_{\mathrm{H}} / R_{\mathrm{H}}$. This is actually the density of ionized hydrogen plus that of neutral hydrogen:

$n_{\mathrm{H}}=n_{\mathrm{p}}+n_{\mathrm{HI}}$.

The size of the ionization zone can then be estimated through the so-called Strömgren radius $r_{\mathrm{S}}$ (e.g. Strömgren 1939), which is defined by equating the rate of ionizing photons $\dot{N}_{\gamma}$ to the number of recombinations per unit time:

$\dot{N}_{\gamma}=\alpha_{\mathrm{rec}}^{(2)} n_{\mathrm{e}}^{2} \frac{4 \pi}{3} r_{\mathrm{S}}^{3}$.

Here, the number density of free electrons is taken to be $n_{\mathrm{e}} \approx n_{\mathrm{H}}$ within the ionized zone, and the rate of ionizing photons is

$\dot{N}_{\gamma} \approx \int_{V} \mathrm{~d} V \int_{E_{\gamma}>13.6 \mathrm{eV}} \mathrm{d} E 4 \pi Q_{\gamma, \mathrm{e}-\mathrm{syn}}$,

which in our cases corresponds mainly to synchrotron radiation of electrons. The recombination coeficient can be taken as $\alpha_{\text {rec }}^{(2)} \approx 2.6 \times 10^{-3} \mathrm{~cm}^{3} \mathrm{~s}^{-1}$ for $T=10^{4} \mathrm{~K}$ and neglecting recombinations to the energy level $n=1$, since this state is ionized again on the spot (e.g. Krolik 1999; Hunt \& Hirashita 2009). It can be checked that in the cases studied here, the Strömgren radius is less than $10 \mathrm{pc}$, which is assumed to be much less than $R_{\mathrm{H}}$. If the ionization fraction is $x=n_{\mathrm{p}} / n_{\mathrm{H}}$, then the density of neutral hydrogen is $n_{\mathrm{H}}(1-x)$, which is an increasing function of the distance within the ionized region. The optical depth at a distance $R$ from the source and along the photon path can be integrated as

$\tau_{\gamma N}\left(E_{\gamma}, R\right)=\int_{0}^{R} \mathrm{~d} l_{\gamma} n_{\mathrm{H}}(1-x) \sigma_{\gamma N}\left(E_{\gamma}\right)$.

According to Petrosian et al. (1972), the fraction of neutral gas reaches unity at the Strömgren radius or even less in the prensence of dust. If the $r_{\mathrm{S}} \gg R_{\mathrm{H}}$ we can approximate the optical depth at $R_{\mathrm{H}}$ as

$\tau_{\gamma N}\left(E_{\gamma}\right) \approx \sigma_{\gamma N}\left(E_{\gamma}\right) N_{\mathrm{H}}$,

which is the expression we use in this work. 


\section{References}

Abdo, A. A., Ackermann, M., Ajello, M., et al. 2009a, ApJ, 707, 1310 Abdo, A. A., Ackermann, M., Ajello, M., et al. 2009b, ApJ, 700, 597 Abdo, A. A., Ackermann, M., Ajello, M., et al. 2009c, ApJ, 707, 55 Abdo, A. A., Ackermann, M., Ajello, M., et al. 2010, ApJ, 719, 1433 Abraham, J., Abreu, P., Aglietta, M., The Pierre Auger Collaboration, et al. 2007, Science, 318, 938

Acciari, V. A., Beilicke, M., Blaylock, G., et al. 2008, ApJ, 679, 397 Acciari, V. A., Aliu, E., Arlen, T., et al. 2009, Science, 325, 444 Aharonian, F. A., Atoian, A. M., \& Nagapetian, A. M. 1983, Astrofizika, 19, 323 Aharonian, F., Akhperjanian, A., Beilicke, M., et al. 2003, A\&A, 403, L1 Aharonian, F., Akhperjanian, A. G., Bazer-Bachi, A. R., et al. 2006, Science, 314,1424

Aharonian, F., Akhperjanian, A. G., Anton, G., et al. 2009, ApJ, 695, L40 Ahrens, J., Bahcall, J. N., Bai, X., et al. 2004, Astrop. Phys., 20, 507 Ajello, M., Costamante, L., Sambruna, R. M., et al. 2009, ApJ, 699, 603 Albert, J., Aliu, E., Anderhub, H., et al. 2008, ApJ, 685, L23 Amsler, C., Doser, M., Antonelli, M., et al. 2008, Phys. Lett. B, 667, 1 Atoyan, A. M., \& Dermer, C. D. 2003, ApJ, 586, 79

Baltz, E. A., Briot, C., Salati, P., Taillet, R., \& Silk, J. 2000, Phys. Rev. D, 61, 023514

Begelman, M. C., Rudak, B., \& Sikora, M. 1990, ApJ, 362, 38 Biretta, J. A., Stern, C. P., \& Harris, D. E. 1991, AJ, 101, 1632 Biretta, J. A., Zhou, F., \& Owen, F. N. 1995, ApJ, 447, 582 Biretta, J. A., Sparks, W. B., \& Macchetto, F. 1999, ApJ, 520, 621 Blumenthal, G. R., \& Gould, R. J. 1970, Rev. Mod. Phys., 42, 237 Bogovalov, S. V., \& Kelner, S. R. 2010, Int. J. Mod. Phys. D, 19, 339 Bond, I. A., Ballet, J., Denis, M., et al. 1996, A\&A, 307, 708 Bosch-Ramon, V., Romero, G. E., \& Paredes, J. M. 2006, A\&A, 447, 263 Böttcher, M. 2007, in The Central Engine of Active Galactic Nuclei, ed. L. C. Ho, \& J.-W. Wang, ASP Conf. Ser., 373, 169

Cappellari, M., Neumayer, N., Reunanen, J., et al. 2009, MNRAS, 175 Cheung, C. C., Harris, D. E., \& Stawarz, Ł. 2007, ApJ, 663, L65

Costamante, L., Aharonian, F., Bühler, R., et al. 2008, in Am. Inst. Phys. Conf. Ser., ed. F. A. Aharonian, W. Hofmann, \& F. Rieger, 1085, 644 Croston, J. H., Kraft, R. P., Hardcastle, M. J., et al. 2009, MNRAS, 395, 1999 Cruddace, R., Paresce, F., Bowyer, S., \& Lampton, M. 1974, ApJ, 187, 497 Dermer, C. D., \& Schlickeiser, R. 2002, ApJ, 575, 667 Despringre, V., Fraix-Burnet, D., \& Davoust, E. 1996, A\&A, 309, 375 Evans, D. A., Kraft, R. P., Worrall, D. M., et al. 2004, ApJ, 612, 786 Fargion, D. 2008, Phys. Scr., 78, 045901

Gaisser, T. K. 1990, Cosmic rays and particle physics, ed. T. K. Gaisser Gebhardt, K., \& Thomas, J. 2009, ApJ, 700, 1690

Gehrels, N., \& Cheung, C. 1992, in Am. Inst. Phys. Conf. Ser., ed. S. S. Holt, S. G. Neff, \& C. M. Urry, 254, 348

Georganopoulos, M., Perlman, E. S., \& Kazanas, D. 2005, ApJ, 634, L33 Ghisellini, G., Haardt, F., Campana, S., Lazzati, D., \& Covino, S. 1999, ApJ, 517,168

Ghisellini, G., Tavecchio, F., \& Chiaberge, M. 2005, A\&A, 432, 401

Ginzburg, V. L., \& Syrovatskii, S. I. 1964, The Origin of Cosmic Rays (New York: Pergamon Press)

Gorbunov, D., Tinyakov, P., Tkachev, I., \& Troitsky, S. 2008, Sov. J. Exp. Theor Phys. Lett., 87, 461

Gould, R. J., \& Schréder, G. P. 1967, Phys. Rev., 155, 1404

Hardcastle, M. J., Cheung, C. C., Feain, I. J., \& Stawarz, Ł. 2009, MNRAS, 393, 1041

Harris, G. L. H., Hesser, J. E., Harris, H. C., \& Curry, P. J. 1984, ApJ, 287, 175 Harris, D. E., Cheung, C. C., Stawarz, Ł., Biretta, J. A., \& Perlman, E. S. 2009, ApJ, 699, 305

Hawarden, T. G., Sandell, G., Matthews, H. E., et al. 1993, MNRAS, 260, 844

Heinz, S. 2008, Int. J. Mod. Phys. D, 17, 1947

Heinz, S., \& Begelman, M. C. 2000, ApJ, 535, 104

Hui, X., Ford, H. C., Ciardullo, R., \& Jacoby, G. H. 1993, ApJ, 414, 463

Hunt, L. K., \& Hirashita, H. 2009, A\&A, 507, 1327

Israel, F. P. 1998, A\&AR, 8, 237

Jokipii, J. R., \& Parker, E. N. 1970, ApJ, 160, 735

Junor, W., Biretta, J. A., \& Livio, M. 1999, Nature, 401, 891

Katarzyński, K., Sol, H., \& Kus, A. 2001, A\&A, 367, 809

Katz, U. F., for the KM3NeT Consortium, \& The KM3NeT Consortium 2009,

Nuclear Instruments and Methods in Physics Research A, 602, 40

Kellermann, K. I., Lister, M. L., Homan, D. C., et al. 2004, ApJ, 609, 539

Kelner, S. R., \& Aharonian, F. A. 2008, Phys. Rev. D, 78, 034013

Kelner, S. R., Aharonian, F. A., \& Bugayov, V. V. 2006, Phys. Rev. D, 74, 034018

Kelner, S. R., Aharonian, F. A., \& Bugayov, V. V. 2009, Phys. Rev. D, 79, 039901

Khangulyan, D., Aharonian, F., \& Bosch-Ramon, V. 2008, MNRAS, 383, 467

Kinzer, R. L., Johnson, W. N., Dermer, C. D., et al. 1995, ApJ, 449, 105
Kirk, J. G., Schneider, P., \& Schlickeiser, R. 1988, ApJ, 328, 269

Komissarov, S. S., Barkov, M. V., Vlahakis, N., \& Königl, A. 2007, MNRAS, 380,51

Kovalev, Y. Y., Lister, M. L., Homan, D. C., \& Kellermann, K. I. 2007, ApJ, 668, L27

Kraft, R. P., Forman, W. R., Jones, C., et al. 2002, ApJ, 569, 54

Kraft, R. P., Kregenow, J. M., Forman, W. R., Jones, C., \& Murray, S. S. 2001, ApJ, 560, 675

Krolik, J. H. 1999, Active galactic nuclei: from the central black hole to the galactic environment (Princeton: Princeton University Press)

Lenain, J.-P., Boisson, C., Sol, H., \& Katarzyński, K. 2008, A\&A, 478, 111

Lieu, R., Mittaz, J. P. D., Bowyer, S., et al. 1996, ApJ, 458, L5

Lipari, P., Lusignoli, M., \& Meloni, D. 2007, Phys. Rev. D, 75, 123005

Ly, C., Walker, R. C., \& Junor, W. 2007, ApJ, 660, 200

Macri, L. M., Huchra, J. P., Stetson, P. B., et al. 1999, ApJ, 521, 155

Marconi, A., Schreier, E. J., Koekemoer, A., et al. 2000, ApJ, 528, 276

Marconi, A., Capetti, A., Axon, D. J., et al. 2001, ApJ, 549, 915

Marscher, A. P., Jorstad, S. G., D’Arcangelo, F. D., et al. 2008, Nature, 452, 966

McKinney, J. C. 2006, MNRAS, 368, 1561

Meier, D. L., Jauncey, D. L., Preston, R. A., et al. 1989, AJ, 98, 27

Mirabel, I. F., Laurent, O., Sanders, D. B., et al. 1999, A\&A, 341, 667

Morganti, R., Oosterloo, T., Struve, C., \& Saripalli, L. 2008, A\&A, 485, L5

Mücke, A., \& Protheroe, R. J. 2001, Astrop. Phys., 15, 121

Mücke, A., Engel, R., Rachen, J. P., Protheroe, R. J., \& Stanev, T. 2000, Comput. Phys. Commun., 124, 290

Mücke, A., Protheroe, R. J., Engel, R., Rachen, J. P., \& Stanev, T. 2003, Astrop. Phys., 18, 593

Neronov, A., \& Aharonian, F. A. 2007, ApJ, 671, 85

Neronov, A., \& Ribordy, M. 2009a, Phys. Rev. D, 80, 083008

Neronov, A., \& Ribordy, M. 2009b, Phys. Rev. D, 79, 043013

Neumayer, N., Cappellari, M., Reunanen, J., et al. 2007, ApJ, 671, 1329

Orellana, M., \& Romero, G. E. 2009, in Am. Inst. Phys. Conf. Ser., ed. C. J. Solano Salinas, J. Bellido, D. Wahl, \& O. Saavedra, 1123, 242

Paciesas, W. S., Harmon, B. A., Wilson, C. A., et al. 1993, in Am. Inst. Phys. Conf. Ser., ed. M. Friedlander, N. Gehrels, \& D. J. Macomb, 280, 473

Pellizza, L. J., Orellana, M., \& Romero, G. E. 2010, Int. J. Mod. Phys. D, 19, 671

Perlman, E. S., Sparks, W. B., Radomski, J., et al. 2001, ApJ, 561, L51

Petrosian, V., Silk, J., \& Field, G. B. 1972, ApJ, 177, L69

Reimer, A., Protheroe, R. J., \& Donea, A. 2004, A\&A, 419, 89

Reynoso, M. M., \& Romero, G. E. 2009, A\&A, 493, 1

Rieger, F. M., \& Aharonian, F. A. 2008a, Int. J. Mod. Phys. D, 17, 1569

Rieger, F. M., \& Aharonian, F. A. 2008b, A\&A, 479, L5

Rieger, F. M., \& Aharonian, F. A. 2009, A\&A, 506, L41

Romero, G. E., \& Vila, G. S. 2008, A\&A, 485, 623

Romero, G. E., Combi, J. A., Perez Bergliaffa, S. E., \& Anchordoqui, L. A. 1996, Astrop. Phys., 5, 279

Rothschild, R. E., Wilms, J., Tomsick, J., et al. 2006, ApJ, 641, 801

Rybicki, G. B., \& Lightman, A. P. 1979, Radiative processes in astrophysics (New York: John Wiley \& Sons)

Ryter, C. E. 1996, Ap\&SS, 236, 285

Sadowski, A., \& Sikora, M. 2010, A\&A, 517, A18

Schlickeiser, R. 2002, Cosmic Ray Astrophysics, ed. R. Schlickeiser

Schlickeiser, R. 2009, MNRAS, 398, 1483

Shi, Y., Rieke, G. H., Hines, D. C., Gordon, K. D., \& Egami, E. 2007, ApJ, 655, 781

Silge, J. D., Gebhardt, K., Bergmann, M., \& Richstone, D. 2005, AJ, 130, 406

Sparks, W. B., Biretta, J. A., \& Macchetto, F. 1996, ApJ, 473, 254

Spitzer, L. 1978, Physical processes in the interstellar medium (New York: John Wiley \& Sons)

Spruit, H. C. 2010, in Lecture Notes in Physics, ed. T. Belloni (Berlin: Springer Verlag), 794, 233

Sreekumar, P., Bertsch, D. L., Hartman, R. C., Nolan, P. L., \& Thompson, D. J. 1999, Astrop. Phys., 11, 221

Steinle, H., Bennett, K., Bloemen, H., et al. 1998, A\&A, 330, 97

Strömgren, B. 1939, ApJ, 89, 526

Tan, J. C., Beuther, H., Walter, F., \& Blackman, E. G. 2008, ApJ, 689, 775

Tavecchio, F., \& Ghisellini, G. 2008, MNRAS, 385, L98

Thompson, D. J., Bertsch, D. L., Dingus, B. L., et al. 1995, ApJS, 101, 259

Torres, D. F., \& Reimer, A. 2011, A\&A, 528, L2

Urry, C. M., \& Padovani, P. 1995, PASP, 107, 803

Vila, G. S., \& Romero, G. E. 2010, MNRAS, 403, 1457

Wardle, M., \& Koenigl, A. 1993, ApJ, 410, 218

Wibig, T., \& Wolfendale, A. W. 2007, Op. Astron. J., 2, 95

Wilson, A. S., \& Yang, Y. 2002, ApJ, 568, 133 